\title{
Compositional modelling of distributed-parameter systems
}

\author{
B.M. Maschke* A.J. van der Schaft ${ }^{\dagger}$
}

\section{Introduction}

The Hamiltonian formulation of distributed-parameter systems has been a challenging reserach area for quite some time. (A nice introduction, especially with respect to systems stemming from fluid dynamics, can be found in [26], where also a historical account is provided.) The identification of the underlying Hamiltonian structure of sets of p.d.e.'s has been instrumental in proving all sorts of results on integrability, the existence of soliton solutions, stability, reduction, etc., and in unifying existing results, see e.g. [11], [24], [18], [17], [25], [14].

Recently, there has been also a surge of interest in the design and control of nonlinear distributed-parameter systems, motivated by various applications. At the same time, it is well-known from finite-dimensional nonlinear control systems [35], [32], [6], [21], [28], [27], [34] a Hamiltonian formulation is helpful in the control design, and the same is to be expected in the distributed-parameter case. However, in extending the theory as for instance exposed in [26] to distributed-parameter control systems a fundamental difficulty arises in the treatment of boundary conditions. Indeed, the treatment of infinite-dimensional Hamiltonian systems in the literature is mostly focussed on systems with infinite spatial domain, where the variables go to zero for the spatial variables tending to infinity, or on systems with boundary conditions such that the energy exchange through the boundary is zero. On the other hand, from a control and interconnection point of view it is quite essential to be able describe a distributed-parameter system with varying boundary conditions inducing energy exchange through the boundary, since in many applications the interaction with the environment (e.g. actuation or measurement) will actually take place through the boundary of the system. Clear examples are the telegraph equations (describing the dynamics of a transmission line), where the boundary of the system is described by the behavior of the voltages and currents at both ends of the transmission line, or a vibrating string (or, more generally, a flexible beam), where it is natural to consider the evolution of the forces and velocities at the ends of the string. Furthermore, in both examples it is obvious that in general the boundary exchange of power (voltage times current in the transmission line example, and force

\footnotetext{
${ }^{*}$ Lab. d'Automatique et de Genie des Procedes, Universite Claude Bernard Lyon-1, F-69622 Villeurbanne, Cedex, France

${ }^{\dagger}$ Dept. of Applied Mathematics, University of Twente, PO Box 217, 7500 AE Enschede, The Netherlands
} 
times velocity for the vibrating string) will be non-zero, and that in fact one would like to consider the voltages and currents or forces and velocities as additional boundary variables of the system, which can be interconnected to other systems. Also for numerical integration and simulation of complex distributed-parameter systems it is essential to be able to describe the complex system as the interconnection or coupling of its subsystems via their boundary variables; for example in the case of coupled fluid-solid dynamics.

From a mathematical point of view, it is not obvious how to incorporate nonzero energy flow through the boundary in the existing Hamiltonian framework for distributed-parameter systems. The problem is already illustrated by the Hamiltonian formulation of e.g. the Korteweg-de Vries equation (see e.g. [26]). Here for zero boundary conditions a Poisson bracket can be formulated with the use of the differential operator $\frac{d}{d x}$, since by integration by parts this operator is obviously skewsymmetric. However, for boundary conditions corresponding to non-zero energy flow the differential operator is not skew-symmetric anymore (since after integrating by parts the remainders are not zero).

In [37], see also [20], we proposed a framework to overcome this fundamental problem by using the notion of a Dirac structure. Dirac structures were originally introduced in [5],[7] as a geometric structure generalizing both symplectic and Poisson structures. Later on (see e.g. [35], [6], [19], [2]) it was realized that in the finitedimensional case Dirac structures can be naturally employed to formalize Hamiltonian systems with constraints as implicit Hamiltonian systems. It turns out that in order to allow the inclusion of boundary variables in distributed-parameter systems the concept of Dirac structure again provides the right type of generalization with respect to the existing framework using Poisson structures.

The Dirac structure for distributed-parameter systems employed in this paper has a specific form by being defined on certain spaces of differential forms on the spatial domain of the system and its boundary, and making use of Stokes' theorem. Its construction emphasizes the geometrical content of the physical variables involved, by identifying them as differential $k$-forms, for appropriate $k$. This interpretation is rather well-known (see e.g. [12]) in the case of Maxwell's equations (and actually directly follows from Faraday's law and Ampère's law), but seems less well-known for the telegraph equations and the description of the Euler's equations for an ideal isentropic fluid.

From the systems and control point of view the approach taken in this paper can be seen as providing the extension of the port-Hamiltonian framework established for lumped-parameter systems in [35], [6], [27], [33], [35], [34], [3] to the distributedparameter case. In the lumped-parameter case this Hamiltonian framework has been successfully employed in the consistent (modular) modeling and simulation of complex interconnected lumped-parameter physical systems, including (actuated) multi-body systems with kinematic constraints and electro-mechanical systems [35], [19], [6], [34], and in the design and control of such systems, exploiting the Hamiltonian and passivity structure in a crucial way [32], [21], [28], [27], [34]. Similar developments can be pursued in the distributed-parameter case; see already [30], [36] for developments in this direction. 
The present paper is organized as follows. In Section 2 we give a general introduction to systems of conservation laws, together with the closure equations relating the conserved quantities to the flux variables. Furthermore, we show how this leads to infinite-dimensional power-continuous interconnection structures and the definition of Hamiltonian functions for energy storage. After this general introduction the main mathematical framework is given in Section 3 and 4, following [37]. In Section 3 it is shown how the notion of a power-continuous interconnection structure as discussed before can be formalized using the geometric concept of a Dirac structure, and in particular the Stokes-Dirac structure. In Section 4 it is shown how this leads to the Hamiltonian formulation of distributed-parameter systems with boundary energy flow, generalizing the notion of finite-dimensional port-Hamiltonian systems. In Section 5 (again following [37]) this is applied to Maxwell's equations on a bounded domain (Subsection 5.1), the telegraph equations for an ideal transmission line (Subsection 5.2), and the vibrating string (Subsection 5.3). Furthermore, by modifying the Stokes-Dirac structure with an additional term corresponding to three-dimensional convection, Euler's equations for an ideal isentropic fluid are studied in Section 6. Section 7 treats the basic notions of Casimir functions determined by the Stokes-Dirac structure. This can be seen as a starting point for control by interconnection of distributed-parameter port-Hamiltonian systems. Finally, Section 8 contains the conclusions.

\section{Systems of two physical domains in canonical inter- action}

The aim of this section is to introduce a class of infinite-dimensional physical systems and to show how they can be represented as port-Hamiltonian systems defined with respect to a special type of infnite-dimensional Dirac structure, called Stokes-Dirac structure. This will be done by formulating the distributed-parameter system as a system of conservation laws [10] [31], each describing the balance equation associated with some conserved physical quantity, coupled with a set of closure equations. These balance laws will define the Stokes-Dirac structure, while the closure equations will turn out to be equivalent with the definition of the Hamiltonian of the system.

\subsection{Conservation laws, interdomain coupling and boundary energy flows: motivational examples}

In this paragraph we shall introduce the main concepts of conservation law, interdomain coupling and boundary energy flow by means of three simple and classical examples of distributed-parameter systems.

The first example is the simplest one, and consists of only one conservation law on a one-dimensional spatial domain. With the aid of this simple example we shall introduce the notions of conservation law, balance equation, variational derivative, finally leading to the definition of a port-Hamiltonian system. 
Example 2.1 (The inviscid Burger's equation). The viscous Burger's equation is a scalar parabolic equation which represents the simplest model for a fluid flow (often used as a numerical test for the asymptotic theory of the Navier-Stokes equations) [31]. It is defined on a one-dimensional spatial domain (an interval) $Z=[a, b] \subset \mathbb{R}$, while its state variable is $\alpha(z, t) z \in Z, t \in I$, where $I$ is an interval of $\mathbb{R}$ satisfying the partial differential equation

$$
\frac{\partial \alpha}{\partial t}+\alpha \frac{\partial \alpha}{\partial z}-\nu \frac{\partial^{2} \alpha}{\partial z^{2}}=0
$$

In the following we shall consider the inviscid Burger's equations (corresponding to the case $\nu=0$ ), which may be alternatively expressed by the following conservation law:

$$
\frac{\partial \alpha}{\partial t}+\frac{\partial}{\partial z} \beta=0
$$

where the state variable $\alpha(z, t)$ is called the conserved quantity and the function $\beta(z, t)$ is called the flux variable and is given by $\beta=\frac{\alpha^{2}}{2}$. Indeed, integrating the partial differential equation (2) for $\nu=0$ on the interval $Z$, one obtains the following balance equation:

$$
\frac{d}{d t} \int_{a}^{b} \alpha d z=\beta(a)-\beta(b)
$$

Furthermore, according to the framework of Irreversible Thermodynamics [29], one may express the flux $\beta$ as a function of the generating force which is the variational derivative (or, functional derivative, ) of some generating functional $H(\alpha)$ of the state variable. This variational derivative plays the same role as the gradient of a function when considering functionals instead of functions. The variational derivative $\frac{\delta H}{\delta \alpha}$ of the functional $H(\alpha)$ is uniquely defined by the requirement:

$$
H(\alpha+\epsilon \eta)=H(\alpha)+\epsilon \int_{a}^{b} \frac{\delta H}{\delta \alpha} \eta d z+O\left(\epsilon^{2}\right)
$$

for any $\epsilon \in \mathbb{R}$ and any smooth function $\eta(z, t)$ such that $\alpha+\epsilon \eta$ satisfies the same boundary conditions as $\alpha$ [26]. For the inviscid Burger's equation it is easy to see that $\beta=\frac{\alpha^{2}}{2}$ can be expressed as $\beta=\frac{\delta H}{\delta \alpha}$, where

$$
H(\alpha)=\int_{a}^{b} \frac{\alpha^{3}}{6} d z
$$

Hence the inviscid Burger's equation may be also expressed as

$$
\frac{\partial \alpha}{\partial t}=-\frac{\partial}{\partial z} \frac{\delta H}{\delta \alpha}
$$

This defines an infinite-dimensional Hamiltonian system [26] with respect to the skew-symmetric operator $\frac{\partial}{\partial z}$ (defined on the functions with support strictly contained in the interval $Z$ ). 
From this formulation one immediately derives that the Hamiltonian $H(\alpha)$ is another conserved quantity. Indeed, by integration by parts

$$
\frac{d}{d t} H=\int_{a}^{b} \frac{\delta H}{\delta \alpha} \cdot \frac{\partial \alpha}{\partial t} d z=\int_{a}^{b} \frac{\delta H}{\delta \alpha} \cdot-\frac{\partial}{\partial z} \frac{\delta H}{\delta \alpha} d z=\left(\beta^{2}(a)-\beta^{2}(b)\right)
$$

Here it is worth to notice that the time variation of the Hamiltonian functional is a quadratic function of the flux variables evaluated at the boundaries of the spatial domain $Z$.

The second example, the p-system, is a classical example that we shall use in order to introduce the concept of an infinite-dimensional port-Hamiltonian system. It corresponds to the case of two physical domains in interaction and consists of a system of two conservations laws.

Example 2.2 (The p-system). The p-system is a model for a 1-dimensional isentropic gas dynamics in Lagrangian coordinates. The independent variable $z$ belong to an interval $Z \subset \mathbb{R}$, It is defined with the following variables: the specific volume $v(z, t) \in \mathbb{R}^{+}$, the velocity $u(z, t)$ and the pressure functional $p(v)$ (which is for instance in the case of a polytropic isentropic ideal gas given by $p(v)=A v^{-\gamma}$ where $\gamma \geq 1$ ). The $p$-system is then defined by the following system of partial differential equations:

$$
\begin{gathered}
\frac{\partial v}{\partial t}-\frac{\partial u}{\partial z}=0 \\
\frac{\partial u}{\partial t}+\frac{\partial p(v)}{\partial z}=0
\end{gathered}
$$

representing the conservation of mass and of momentum. By defining the state vector as: $\alpha(z, t)=\left(\begin{array}{c}\alpha_{1} \\ \alpha_{2}\end{array}\right)=\left(\begin{array}{c}v \\ u\end{array}\right)$ and the vector valued flux $\beta(z, t)=\left(\begin{array}{c}\beta_{1} \\ \beta_{2}\end{array}\right)=$ $\left(\begin{array}{c}-u \\ p(v)\end{array}\right)$ the p-system is rewritten as

$$
\frac{\partial \alpha}{\partial t}+\frac{\partial}{\partial z} \beta=0
$$

Again, according to the framework of Irreversible Thermodynamics, the flux variables may be written as functions of the variational derivatives of some generating functionals. Consider the functional $H(\alpha)=\int_{a}^{b} \mathcal{H}(v, u) d z$ where $\mathcal{H}(v, u)$ denotes the energy density, which is given as the sum of the internal energy and the kinetic energy densities

$$
\mathcal{H}(v, u)=\mathcal{U}(v)+\frac{u^{2}}{2}
$$

where $-\mathcal{U}(v)$ is a primitive function of the pressure. Note that the expression of the kinetic energy does not depend on the mass density which is assumed to be constant and for simplicity is set equal to 1 . Hence no difference is made between the velocity and the momentum. The vector of fluxes $\beta$ may now be expressed in term of the generating forces as follows

$$
\beta=\left(\begin{array}{c}
-\frac{\delta H}{\delta u} \\
-\frac{\delta H}{\delta v}
\end{array}\right)=\left(\begin{array}{cc}
0 & -1 \\
-1 & 0
\end{array}\right)\left(\begin{array}{l}
\frac{\delta H}{\delta v} \\
\frac{\delta H}{\delta u}
\end{array}\right)
$$


The anti-diagonal matrix represents the canonical coupling between two physical domains: the kinetic and the potential (internal) domain (for lumped parameter systems this is discussed e.g. in [4]). The variational derivative of the total energy with respect to the state variable of one domain generates the flux variable for the other domain.

Combining the equations (9) and (11), the p-system may thus be written as the following Hamiltonian system:

$$
\frac{\partial \alpha}{\partial t}=\left(\begin{array}{cc}
0 & -\frac{\partial}{\partial z} \\
-\frac{\partial}{\partial z} & 0
\end{array}\right)\left(\begin{array}{l}
\frac{\delta H}{\delta \alpha_{1}} \\
\frac{\delta H}{\delta \alpha_{2}}
\end{array}\right)
$$

From the Hamiltonian form of the system and using again integration by parts, one may derive that the total energy obeys the following power balance equation:

$$
\frac{d}{d t} H=\beta_{1}(a) \beta_{2}(a)-\beta_{1}(b) \beta_{2}(b)
$$

Notice again that the right-hand side of this power-balance equation is a quadratic function of the fluxes at the boundary of the spatial domain.

Remark 2.3. It is important to note that any non-linear wave equation:

$$
\frac{\partial^{2} g}{\partial t^{2}}-\frac{\partial}{\partial z}\left(\sigma\left(\frac{\partial g}{\partial z}\right)\right)=0
$$

may be expressed as a p-system using the change of variables $u=\frac{\partial g}{\partial t}, v=\frac{\partial g}{\partial z}$ and $p(v)=-\sigma(v)$.

The last example is the vibrating string. Actually it is again a system of two conservation laws representing the canonical interdomain coupling between the kinetic energy and the elastic potential energy. However in this example, unlike the p-system, the classical choice of the state variables leads to express the total energy as a function of some of the spatial derivatives of the state variables. We shall analyze how the dynamic equations and the power balance are expressed in this case and we shall subsequently draw some conclusions on the choice of the state variables.

Example 2.4 (Vibrating string). Consider an elastic string subject to traction forces at its ends. The spatial variable $z$ belongs to the interval $Z=[a, b] \subset \mathbb{R}$. Denote by $u(t, z)$ the displacement of the string and the velocity by $v(z, t)=\frac{\partial u}{\partial t}$. Using the vector of state variables $x(z, t)=(u, v)^{T}$, the dynamics of the vibrating string is described by the system of partial differential equations

$$
\frac{\partial x}{\partial t}=\left(\begin{array}{c}
v \\
\frac{1}{\mu} \frac{\partial}{\partial z}\left(T \frac{\partial u}{\partial z}\right)
\end{array}\right)
$$

where the first equation is simply the definition of the velocity and the second one is Newton's second law.

The time variation of the state may be expressed as a function of the variational derivative of the total energy as in the preceeding examples. Indeed, define the total 
energy as $H(x)=U(u)+K(v)$, where $U$ denotes the elastic potential energy and $K$ the kinetic energy of the string. The elastic potential energy is given as a function of the $\operatorname{strain} \epsilon(t, z)=\frac{\partial u}{\partial z}$

$$
U(u)=\int_{a}^{b} \frac{1}{2} T\left(\frac{\partial u}{\partial z}\right)^{2} d z
$$

with $T$ the elasticity modulus. The kinetic energy $K$ is the following function of the velocity $v(z, t)=\frac{\partial u}{\partial t}$

$$
K(v)=\int_{a}^{b} \frac{1}{2} \mu v(z, t)^{2} d z
$$

Thus the total system (14) may be expressed as

$$
\frac{\partial x}{\partial t}=\left(\begin{array}{cc}
0 & \frac{1}{\mu} \\
-\frac{1}{\mu} & 0
\end{array}\right)\left(\begin{array}{c}
\frac{\delta H}{\delta u} \\
\frac{\delta H}{\delta v}
\end{array}\right)
$$

where according to the definition of the variational derivative given in (4) one obtains

$$
\frac{\delta H}{\delta u}=\frac{\delta U}{\delta u}=-\frac{\partial}{\partial z}\left(T \frac{\partial u}{\partial z}\right)
$$

which is the elastic force and

$$
\frac{\delta H}{\delta v}=\frac{\delta K}{\delta v}=\mu v
$$

which is the momentum.

In the formulation of equation (17) there appears again an anti-diagonal skewsymmetric matrix which corresponds to the expression of a canonical interdomain coupling between the elastic energy domain and the kinetic energy domain. However the system is not expressed as a system of conservation laws since the rate of change of the state variables is a linear combination of the variational derivatives directly (and not of their spatial derivatives). Instead of being a simplification, this reveals a drawback for the case that there is energy flow through the boundary of the spatial domain. Indeed in this case, the variational derivative has to be completed by a boundary term since the Hamiltonian functional depends on the spatial derivatives of the state. For the elastic potential energy this becomes (integration by parts)

$$
U(u+\epsilon \eta)=U(u)-\epsilon \int_{a}^{b} \frac{\partial}{\partial z}\left(T \frac{\partial u}{\partial z}\right) \eta d z+\epsilon\left[\eta\left(T \frac{\partial u}{\partial z}\right)\right]_{a}^{b}+O\left(\epsilon^{2}\right)
$$

On the other hand, writing the system (14) as a second order equation yields the wave equation

$$
\mu \frac{\partial^{2} u}{\partial t^{2}}=\frac{\partial}{\partial z}\left(T \frac{\partial u}{\partial z}\right)
$$

which according to Remark 2.3 may be alternatively expressed as a p-system. 
In the sequel we shall formulate the vibrating string as a system of two conservation laws, which is however slightly different from the p-system formulated before. It differs from the p-system by the choice of the state variables in such a way that, first, the mass density may depend on the spatial variable $z$ (which is not the case in the Hamiltonian density function defined in equation (10)), and secondly, that the variational derivatives of the total energy equal the co-energy variables.

Indeed, we take as vector of state variables

$$
\alpha(z, t)=\left(\begin{array}{c}
\epsilon \\
p
\end{array}\right)
$$

where $\epsilon$ denotes the strain $\alpha_{1}=\epsilon=\frac{\partial u}{\partial z}$ and $p$ denotes the momentum $\alpha_{2}=p=\mu v$. Recall that in these variables the total energy is written as

$$
H_{0}=\int_{a}^{b} \frac{1}{2}\left(T \alpha_{1}^{2}+\frac{1}{\mu} \alpha_{2}^{2}\right) d z
$$

Notice that the energy functional now only depends on the state variables and not on their spatial derivatives. Furthermore, one may define the flux variables to be the stress $\beta_{1}=\frac{\delta H_{0}}{\delta \alpha_{1}}=T \alpha_{1}$ and the velocity $\beta_{2}=\frac{\delta H_{0}}{\delta \alpha_{1}}=\frac{\alpha_{2}}{\mu}$. In matrix notation, the fluxes are expressed as a function of the generating forces $\frac{\delta H_{0}}{\delta \alpha}$ by:

$$
\beta=\left(\begin{array}{c}
-\frac{\partial H_{0}}{\partial \epsilon} \\
-\frac{\partial H_{0}}{\partial p}
\end{array}\right)=\left(\begin{array}{cc}
0 & -1 \\
-1 & 0
\end{array}\right)\left(\begin{array}{l}
\frac{\delta H_{0}}{\delta \alpha_{1}} \\
\frac{\delta H_{0}}{\delta \alpha_{2}}
\end{array}\right)=\left(\begin{array}{cc}
0 & -1 \\
-1 & 0
\end{array}\right) \frac{\delta H_{0}}{\delta \alpha}
$$

Thus the model of the vibrating string may be expressed by the system of two conservation laws (as for the p-system):

$$
\frac{\partial \alpha}{\partial t}=\left(\begin{array}{cc}
0 & \frac{\partial}{\partial z} \\
\frac{\partial}{\partial z} & 0
\end{array}\right) \frac{\delta H_{0}}{\delta \alpha}
$$

which satisfies also the power balance equation (13).

\subsection{Systems of two conservation laws in canonical interaction}

In this section we shall consider the general class of distributed-parameter systems consisting of two conservation laws with the canonical coupling presented as in the above examples of the p-system and the vibrating string. In the first part, for 1dimensional spatial domains, we shall introduce the concept of interconnection structure and port variables which are fundamental to the definition of port-Hamiltonian systems. On this case we shall also introduce the notion of differential forms. In the second part we shall give the definition of systems of two conservation laws defined on $n$-dimensional spatial domains. We do not use the usual vector calculus formulation but express the systems in terms of differential forms [1] [16]. This leads to concise, coordinate independent formulations and unifies the notations for the various physical domains. 


\subsubsection{Interconnection structure, boundary energy flows and port-based formulation for 1-D spatial domains}

\section{Interconnection structure and power continuity}

Let us consider the systems of two conservation laws arising from the modelling of two physical domains in canonical interaction as have been presented for the vibrating string and the p-system:

$$
\frac{\partial \alpha}{\partial t}=\left(\begin{array}{cc}
0 & \frac{\partial}{\partial z} \\
\frac{\partial}{\partial z} & 0
\end{array}\right) \frac{\delta H_{0}}{\delta \alpha}
$$

where $\alpha=\left(\alpha_{1}(z, t), \alpha_{2}(z, t)\right)^{T}$. Let us now define an interconnection structure for this system in the sense of network [13] [4] or port-based modelling [23] [35]. Define the vector of flow variables to be the time variation of the state and denote it by:

$$
f=\frac{\partial \alpha}{\partial t}
$$

Define the vector of effort variables $e$ to be the vector of the generating forces given as

$$
e=\frac{d H_{0}}{d \alpha}
$$

The flow and effort variables are power-conjugated since their product is the timevariation of the total energy:

$$
\frac{d}{d t} H_{0}=\int_{a}^{b}\left(\frac{\partial \mathcal{H}_{0}}{\partial \alpha_{1}} \frac{\delta \alpha_{1}}{\delta t}+\frac{\partial \mathcal{H}_{0}}{\partial \alpha_{2}} \frac{\delta \alpha_{2}}{\delta t}\right) d z=\int_{a}^{b}\left(e_{1} f_{1}+e_{2} f_{2}\right) d z
$$

where $\mathcal{H}_{0}$ denotes the density corresponding to $H_{0}$. Considering the right-hand side of the power balance equation (13) it is clear that the energy exchange of the system with its environment is determined by the flux variables restricted to the boundary of the domain. Therefore let us define two external boundary variables as follows:

$$
\left(\begin{array}{c}
f_{\partial} \\
e_{\partial}
\end{array}\right)=\left(\begin{array}{c}
e_{2} \\
e_{1}
\end{array}\right)=\left(\begin{array}{c}
\frac{\delta H_{0}}{\delta \alpha_{2}} \\
\frac{\delta H_{0}}{\delta \alpha_{1}}
\end{array}\right)=\left(\begin{array}{c}
v \\
\sigma
\end{array}\right)
$$

These boundary variables are also power-conjugated as their product $\beta_{1} \beta_{2}=e_{b} f_{b}=$ $\sigma v$ equals the right-hand side of the power balance equation (13). Considering the four power-conjugated variables $f_{1}, f_{2}, f_{\partial}, e_{1}, e_{2}, e_{\partial}$, the power balance equation (13) implies that their product is zero:

$$
\int_{a}^{b}\left(e_{1} f_{1}+e_{2} f_{2}\right) d z+e_{\partial}(b) f_{\partial}(b)-e_{\partial}(a) f_{\partial}(a)=0
$$

This bilinear product between the power-conjugated variables is analogous to the product between the circuit variables expressing the power continuity relation in circuits and network models [13] [4]. Such products (or pairings are also central in the definition of implicit Hamiltonian systems [5] [7] and port-Hamiltonian systems 
in finite dimensions [35] [19]. In the forthcoming sections we shall show that this product will play the same role for infinite-dimensional port-Hamiltonian systems [20] [37].

The interconnection structure underlying the system (26) (analogous to Kirchhoff's laws for circuits) may now be summarized by (30) together with

$$
f=\left(\begin{array}{cc}
0 & \frac{\partial}{\partial z} \\
\frac{\partial}{\partial z} & 0
\end{array}\right) e
$$

\section{Introduction to differential forms}

Let us now introduce for the case of the 1-dimensional spatial domain the use of differential forms in the formulation of systems of conservation laws. Until now we have simply considered the state variables $\alpha$ and the flux variables $\beta$ as functions on the space-time domain $Z \times I$. However considering the balance equation (3)

$$
\frac{d}{d t} \int_{a}^{b} \alpha d z=\beta(a)-\beta(b)
$$

associated with the conservation law (2) it becomes clear that they are of different nature. The state variables $\alpha$ correspond to conserved quantities through integration, while the flux variables $\beta$ correspond to functions which can be evaluated at any point (for instance at the boundary points of the spatial domain). This distinction may be expressed by representing the variables as differential forms. For the case of one-dimensional spatial domains considered in this paragraph, the state variables are identified with differential forms of degree 1 , which can be integrated along one-dimensional curves. The flux variables, on the other hand, are identified with differential forms of degree 0 , that means functions evaluated at points of the spatial domain. The reader is referred to the following textbooks [1] [12] [16] for an exhaustive definition of differential forms that we shall use systematically in the rest of the paper.

\subsubsection{Interconnection structure, boundary energy flows and port-based formulation for $\mathbf{n}$-dimensional spatial domains}

\section{Systems of two conservation laws with canonical interdomain coupling}

In this paragraph we shall give the general definition of the class of systems of conservation laws that we shall consider in the forthcoming sections. We first recall the expression of systems of conservation laws defined on $n$-dimensional spatial domains, and secondly generalize the systems of two conservation laws with canonical interdomain coupling as defined in the previous section 2.2 to the $n$-dimensional spatial domain.

Define the spatial domain of the considered distributed-parameter system as $Z \in \mathbb{R}^{n}$ being an $n$-dimensional smooth manifold with smooth $(n-1)$-dimensional boundary $\partial Z$. Denote by $\Omega^{k}(Z)$ the vector space of (differential) $k$-forms on $Z$ (respectively by $\Omega^{k}(\partial Z)$ the vector space of $k$-forms on $\partial Z$ ). Denote furthermore $\Omega=\bigoplus_{k \geq 0} \Omega^{k}(Z)$ the algebra of differential forms over $Z$ and recall that it is endowed with an exterior product $\wedge$ and an exterior derivation $d$ [1] [16]. 
Definition 2.5. A system of conservation laws is defined by a set of conserved quantities $\alpha_{i} \in \Omega^{k_{i}}(Z), i \in\{1, \ldots, N\}$ where $N \in \mathbb{N}, k_{i} \in \mathbb{N}$, defining the state space $\mathcal{X}=\bigotimes_{i=1, . ., N} \Omega^{k_{i}}(Z)$. They satisfy a set of conservation laws

$$
\frac{\partial \alpha_{i}}{\partial t}+\mathrm{d} \beta_{i}=g_{i}
$$

where $\beta_{i} \in \Omega^{k_{i}-1}(Z)$ denote the set of fluxes and $g_{i} \in \Omega^{k_{i}}(Z)$ denote the set of distributed interaction forms. Finally, the fluxes $\beta_{i}$ are defined by the closure equations

$$
\beta_{i}=J\left(\alpha_{i}, z\right), i=1, . ., N
$$

The integral form of the conservation laws yield the following balance equations

$$
\frac{d}{d t} \int_{Z} \alpha_{i}+\int_{\partial Z} \beta_{i}=\int_{Z} g_{i}
$$

Remark 2.6. A common case is that the conserved quantities are 3 -forms, that is, the balance equation is evaluated on volumes of the 3 -dimensional space. Then, in vector calculus notation, the conserved quantities may be identified with vectors $u_{i}$ on $Z$, the interaction terms $g_{i}$ may also be considered as vectors, and the fluxes may be identified with vectors $q_{i}$. In this case the system of conservation laws takes the more familiar form:

$$
\frac{\partial u_{i}}{\partial t}(z, t)+\operatorname{div}_{z} q_{i}=g_{i}, i=1, . ., n
$$

However, systems of conservation laws may correspond to differential forms of any degree. Maxwell's equations provide a classical example where the conserved quantities are actually differential forms of degree 3 [12].

In the sequel, as in the case of the 1-dimensional spatial domain, we shall consider a particular class of systems of conservation laws where the fluxes, determined by the closure equations, are (linear) functions of the derivatives of some generating function. One may note again that this is in agreement with the general assumptions of irreversible thermodynamics [29] where the flux variables are (eventually nonlinear) functions of the generating forces, being the derivative of some generating functional. More precisely, we shall consider closure equations arising from the description of the canonical interaction of two physical domains (for instance the kinetic and elastic energy in the case of the vibrating string, or the electric and magnetic energy for electromagnetic fields) [20].

First recall the general definition of the variational derivative of a functional $H(\alpha)$ with respect to the differential form $\alpha \in \Omega^{p}(Z)$ (generalizing the definition given before).

Definition 2.7. Consider a density function $\mathcal{H}: \Omega^{p}(Z) \times Z \rightarrow \Omega^{n}(Z)$ where $p \in$ $\{1, . ., n\}$, and denote by $H:=\int_{Z} \mathcal{H} \in \mathbb{R}$ the associated functional. Then the uniquely defined differential form $\frac{\delta H}{\delta \alpha} \in \Omega^{n-p}(Z)$ which satisfies for all $\Delta \alpha \in \Omega^{p}(Z)$ and $\varepsilon \in \mathbb{R}$ :

$$
H(\alpha+\varepsilon \Delta \alpha)=\int_{Z} \mathcal{H}(\alpha+\varepsilon \Delta \alpha)=\int_{Z} \mathcal{H}(\alpha)+\varepsilon \int_{Z}\left[\frac{\delta H}{\delta \alpha} \wedge \Delta \alpha\right]+\mathrm{O}\left(\varepsilon^{2}\right)
$$

is called the variational derivative of $H$ with respect to $\alpha \in \Omega^{p}(Z)$. 
Now we define the generalization of the systems presented in the section 2.2 to spatial domains of arbitrary dimension.

Definition 2.8. Systems of two conservation laws with canonical interdomain coupling are systems of two conservation laws involving a pair of conserved quantities $\alpha_{p} \in \Omega^{p}(Z)$ and $\alpha_{q} \in \Omega^{q}(Z)$, differential forms on the $n$-dimensional spatial domain $Z$ of degree $p$ and $q$ respectively, where the integers $p$ and $q$ satisfy $p+q=n+1$. The closure equations generated by a Hamiltonian density function $\mathcal{H}: \Omega^{p}(Z) \times \Omega^{q}(Z) \times Z \rightarrow \Omega^{n}(Z)$ resulting in the total Hamiltonian $H:=\int_{Z} \mathcal{H} \in \mathbb{R}$ are given by:

$$
\left(\begin{array}{c}
\beta_{p} \\
\beta_{q}
\end{array}\right)=\varepsilon\left(\begin{array}{cc}
0 & (-1)^{r} \\
1 & 0
\end{array}\right)\left(\begin{array}{c}
\frac{\delta H}{\delta \alpha_{p}} \\
\frac{\delta H}{\delta \alpha_{q}}
\end{array}\right)
$$

where $r=p q+1, \varepsilon \in\{-1,+1\}$ depending on the sign convention of the considered physical domain.

Remark 2.9. The total Hamiltonian $H\left(\alpha_{q}, \alpha_{p}\right)$ corresponds to the energy function of a physical system, the state variables $\alpha_{i}$ are called the energy variables and the variational derivatives $\frac{\delta H}{\delta \alpha_{i}}$ are called the co-energy variables.

\section{Boundary port variables and the power continuity relation}

In the same way as for systems defined on 1-dimensional spatial domains, one may define for $n$ - spatial domains pairs of power conjugated variables. Define the flow variables to be the time-variation of the state denoted by

$$
\left(\begin{array}{c}
f_{p} \\
f_{q}
\end{array}\right)=\left(\begin{array}{c}
\frac{\partial \alpha_{p}}{\partial t} \\
\frac{\partial \alpha_{q}}{\partial t}
\end{array}\right)
$$

Furthermore, define the vector of effort variables to be the vector of the generating forces denoted by

$$
\left(\begin{array}{c}
e_{p} \\
e_{q}
\end{array}\right)=\left(\begin{array}{c}
\frac{\delta H}{\delta \alpha_{p}} \\
\frac{\delta H}{\delta \alpha_{q}}
\end{array}\right)
$$

The flow and effort variables are power-conjugated as their product is the timevariation of the Hamiltonian function:

$$
\frac{d H}{d t}=\int_{Z}\left(\frac{\delta H}{\delta \alpha_{p}} \wedge \frac{\partial \alpha_{p}}{\partial t}+\frac{\delta H}{\delta \alpha_{q}} \wedge \frac{\partial \alpha_{q}}{\partial t}\right)=\int_{Z}\left(e_{p} \wedge f_{p}+e_{q} \wedge f_{q}\right)
$$

Using the conservation laws (36), the closure relations (37) and the properties of the exterior derivative and Stokes' theorem, one may write the time-variation of the Hamiltonian as

$$
\begin{aligned}
\frac{d H}{d t} & =\int_{Z}\left(\varepsilon \beta_{q} \wedge\left(-d \beta_{p}\right)+(-1)^{r} \beta_{p} \wedge \varepsilon\left(-d \beta_{q}\right)\right) \\
& =-\varepsilon \int_{Z}\left(\beta_{q} \wedge d \beta_{p}+(-1)^{p q+1}(-1)^{(p-1) q} \beta_{q} \wedge d \beta_{p}\right) \\
& =-\varepsilon \int_{Z}\left(\beta_{q} \wedge d \beta_{p}+(-1)^{q} \beta_{q} \wedge d \beta_{p}\right) \\
& =-\varepsilon \int_{\partial Z} \beta_{q} \wedge \beta_{p}
\end{aligned}
$$


Finally we define flow and effort variables on the boundary of the system as the restriction of the flux variables to the boundary $\partial Z$ of the domain $Z$ :

$$
\left(\begin{array}{c}
f_{\partial} \\
e_{\partial}
\end{array}\right)=\left(\begin{array}{c}
\left.\beta_{q}\right|_{\partial Z} \\
\left.\beta_{p}\right|_{\partial Z}
\end{array}\right)
$$

They are also power conjugated variables as their product defined in (42) is the time variation of the Hamiltonian functional (the total energy of the physical system).

On the total space of power-conjugated variables, the differential forms $\left(f_{p}, e_{p}\right)$ and $\left(f_{q}, e_{q}\right)$ on the domain $Z$ and the differential forms $\left(f_{\partial}, e_{\partial}\right)$ defined on the boundary $\partial Z$, one may define an interconnection structure, underlying the system of two conservation laws with canonical interdomain coupling of Definition 2.8. This interconnection structure is defined by the equation (42) together with (combining the conservation laws (36) with the closure equation (37))

$$
\left(\begin{array}{c}
f_{q} \\
f_{p}
\end{array}\right)=\varepsilon\left(\begin{array}{ccc}
0 & (-1)^{r} & d \\
d & 0
\end{array}\right)\left(\begin{array}{l}
e_{q} \\
e_{p}
\end{array}\right)
$$

This interconnection is power-continuous in the sense that the power-conjugated variables related by (42) and (43) satisfy the power continuity relation:

$$
\int_{Z}\left(e_{p} \wedge f_{p}+e_{q} \wedge f_{q}\right)+\varepsilon \int_{\partial Z} f_{\partial} \wedge e_{\partial}=0
$$

This expression is the straightforward consequence of the two expressions of the variation of the Hamiltonian $H$ in (40) (41).

In the next sections 3 and 4 we shall show how the above power-continuous interconnection structure can be formalized as geometric structure, called Dirac structure, and how this leads to the definion of infinite-dimensional Hamiltonian systems with energy flows at the boundary of their spatial domain, called portHamiltonian systems.

\section{Stokes-Dirac structures}

\subsection{Dirac structures}

The notion of a Dirac structure was originally introduced in [5], [7] as a geometric structure generalizing both symplectic and Poisson structures. In e.g. [35], [19], [33], [2], [6], [34], [3], it was employed as the geometrical notion formalizing general power-conserving interconnections, thereby allowing the Hamiltonian formulation of interconnected and constrained mechanical and electrical systems.

A definition of Dirac structures (which is actually slightly more general than the one in [5], [7]) can be given as follows. Let $\mathcal{F}$ and $\mathcal{E}$ be linear spaces, equipped with a pairing, that is, a bilinear operation

$$
\mathcal{F} \times \mathcal{E} \rightarrow L
$$

with $L$ a linear space. The pairing will be denoted by $\langle e \mid f\rangle \in L, f \in \mathcal{F}, e \in \mathcal{E}$. By symmetrizing the pairing we obtain a symmetric bilinear form $\ll$, 》 on $\mathcal{F} \times \mathcal{E}$, with values in $L$, defined as

$$
\ll\left(f_{1}, e_{1}\right),\left(f_{2}, e_{2}\right) \gg:=<e_{1}\left|f_{2}>+<e_{2}\right| f_{1}>, \quad\left(f_{i}, e_{i}\right) \in \mathcal{F} \times \mathcal{E}
$$


Definition 3.1. Let $\mathcal{F}$ and $\mathcal{E}$ be linear spaces with a pairing $<\mid>$. A Dirac structure is a linear subspace $D \subset \mathcal{F} \times \mathcal{E}$ such that $D=D^{\perp}$, with $\perp$ denoting the orthogonal complement with respect to the bilinear form $\ll, \gg$.

Example 3.2. Let $\mathcal{F}$ be a linear space over $\mathbb{R}$. Let $\mathcal{E}$ be given as $\mathcal{F}^{*}$ (the space of linear functionals on $\mathcal{F}$ ), with pairing \langle|$>$ the duality product $\langle e \mid f\rangle \in \mathbb{R}$.

(a) Let $J: \mathcal{E} \rightarrow \mathcal{F}$ be a skew-symmetric map. Then graph $J \subset \mathcal{F} \times \mathcal{E}$ is a Dirac structure.

(b) Let $\omega: \mathcal{F} \rightarrow \mathcal{E}$ be a skew-symmetric map. Then graph $\omega \subset \mathcal{F} \times \mathcal{E}$ is a Dirac structure.

(c) Let $V \subset \mathcal{F}$ be a finite-dimensional linear subspace. Then $V \times V^{\text {orth }} \subset \mathcal{F} \times \mathcal{E}$ is a Dirac structure, where $V^{\text {orth }} \subset \mathcal{E}$ is the annihilating subspace of $V$. The same holds if $\mathcal{F}$ is a topological vectorspace, $\mathcal{E}$ is the space of linear continuous functionals on $\mathcal{F}$, and $V$ is a closed subspace of $\mathcal{F}$.

Example 3.3. Let $M$ be a finite-dimensional manifold. Let $\mathcal{F}=V(M)$ denote the Lie algebra of smooth vector fields on $M$, and let $\mathcal{E}=\Omega^{1}(M)$ be the linear space of smooth 1-forms on $M$. Consider the usual pairing $\langle\alpha \mid X\rangle=i_{X} \alpha$ between 1-forms $\alpha$ and vectorfields $X$; implying that $L$ is the linear space of smooth functions on $M$.

(a) Let $J$ be a Poisson structure on $M$, defining a skew-symmetric mapping $J$ : $\Omega^{1}(M) \rightarrow V(M)$. Then graph $J \subset V(M) \times \Omega^{1}(M)$ is a Dirac structure.

(b) Let $\omega$ be a (pre-)symplectic structure on $M$, defining a skew-symmetric mapping $\omega: V(M) \rightarrow \Omega^{1}(M)$. Then graph $\omega \subset V(M) \times \Omega^{1}(M)$ is a Dirac structure.

(c) Let $V$ be a constant-dimensional distribution on $M$, and let ann $V$ be its annihilating co-distribution. Then $V \times a n n V$ is a Dirac structure.

Remark 3.4. Usually in Example 3.3 an additional integrability condition is imposed on the Dirac structure, cf. [5], [7]. In part (a) this condition is equivalent to the Jacobi-identity for the Poisson structure; in part (b) it is equivalent to the closedness of the presymplectic structure, while in part (c) it is equivalent to the involutivity of the distribution $D$. Integrability is equivalent to the existence of canonical coordinates, cf. [5], [7], [6]. Various formulations of integrability of Dirac structures and their implications have been worked out in [6]. For the developments of the current paper the notion of integrability is not crucial; see however the comment in the Conclusions.

From the defining property $D=D^{\perp}$ of a Dirac structure it directly follows that for any $(f, e) \in D$

$$
0=\ll(f, e),(f, e) \gg=2<e \mid f>
$$

Thus if $(f, e)$ is a pair of power variables(e.g., currents and voltages in an electric circuit context, or forces and velocities in a mechanical context), then the condition $(f, e) \in D$ implies power-conservation $\langle e \mid f\rangle=0$ (as do Kirchhoff's laws or 
Newton's third law). This is the starting point for the geometric formulation of general power-conserving interconnections in physical systems by Dirac structures as alluded to above.

\subsection{Stokes-Dirac structures}

In this subsection we treat the underlying geometric framework for the Hamiltonian formulation of distributed-parameter systems on a bounded spatial domain, with non-zero energy flow through the boundary. The key concept is the introduction of a special type of Dirac structure on suitable spaces of differential forms on the spatial domain and its boundary, making use of Stokes' theorem. A preliminary treatment of this Dirac structure has been given in [20], [22].

Throughout, let $Z$ be an $n$-dimensional smooth manifold with smooth $(n-1)$ dimensional boundary $\partial Z$, representing the space of spatial variables.

Denote by $\Omega^{k}(Z), k=0,1, \cdots, n$, the space of exterior $k$-forms on $Z$, and by $\Omega^{k}(\partial Z), k=0,1, \cdots, n-1$, the space of $\mathrm{k}$-forms on $\partial Z$. (Note that $\Omega^{0}(Z)$, respectively $\Omega^{0}(\partial Z)$, is the space of smooth functions on $Z$, respectively $\partial Z$.) Clearly, $\Omega^{k}(Z)$ and $\Omega^{k}(\partial Z)$ are (infinite-dimensional) linear spaces (over $\mathbb{R}$ ). Furthermore, there is a natural pairing between $\Omega^{k}(Z)$ and $\Omega^{n-k}(Z)$ given by

$$
<\beta \mid \alpha>:=\int_{Z} \beta \wedge \alpha \quad(\in \mathbb{R})
$$

with $\alpha \in \Omega^{k}(Z), \beta \in \Omega^{n-k}(Z)$, where $\wedge$ is the usual wedge product of differential forms yielding the $n$-form $\beta \wedge \alpha$. In fact, the pairing (48) is non-degenerate in the sense that if $\langle\beta \mid \alpha\rangle=0$ for all $\alpha$, respectively for all $\beta$, then $\beta=0$, respectively $\alpha=0$.

Similarly, there is a pairing between $\Omega^{k}(\partial Z)$ and $\Omega^{n-1-k}(\partial Z)$ given by

$$
<\beta \mid \alpha>:=\int_{\partial Z} \beta \wedge \alpha
$$

with $\alpha \in \Omega^{k}(\partial Z), \beta \in \Omega^{n-1-k}(\partial Z)$. Now let us define the linear space

$$
\mathcal{F}_{p, q}:=\Omega^{p}(Z) \times \Omega^{q}(Z) \times \Omega^{n-p}(\partial Z)
$$

for any pair $p, q$ of positive integers satisfying

$$
p+q=n+1,
$$

and correspondingly let us define

$$
\mathcal{E}_{p, q}:=\Omega^{n-p}(Z) \times \Omega^{n-q}(Z) \times \Omega^{n-q}(\partial Z) .
$$

Then the pairing (48) and (49) yields a (non-degenerate) pairing between $\mathcal{F}_{p, q}$ and $\mathcal{E}_{p, q}$ (note that by $\left.(51)(n-p)+(n-q)=n-1\right)$. As before (see (46)), symmetrization of this pairing yields the following bilinear form on $\mathcal{F}_{p, q} \times \mathcal{E}_{p, q}$ with values in $\mathbb{R}$ :

$$
\begin{aligned}
& \ll\left(f_{p}^{1}, f_{q}^{1}, f_{b}^{1}, e_{p}^{1}, e_{q}^{1}, e_{b}^{1}\right),\left(f_{p}^{2}, f_{q}^{2}, f_{b}^{2}, e_{p}^{2}, e_{q}^{2}, e_{b}^{2}\right) \gg:= \\
& \int_{Z}\left[e_{p}^{1} \wedge f_{p}^{2}+e_{q}^{1} \wedge f_{q}^{2}+e_{p}^{2} \wedge f_{p}^{1}+e_{q}^{2} \wedge f_{q}^{1}\right]+\int_{\partial Z}\left[e_{b}^{1} \wedge f_{b}^{2}+e_{b}^{2} \wedge f_{b}^{1}\right]
\end{aligned}
$$


where for $i=1,2$

$$
\begin{aligned}
& f_{p}^{i} \in \Omega^{p}(Z), f_{q}^{i} \in \Omega^{q}(Z) \\
& e_{p}^{i} \in \Omega^{n-p}(Z), e_{p}^{i} \in \Omega^{n-q}(Z) \\
& f_{b}^{i} \in \Omega^{n-p}(\partial Z), e_{b}^{i} \in \Omega^{n-q}(\partial Z)
\end{aligned}
$$

The spaces of differential forms $\Omega^{p}(Z)$ and $\Omega^{q}(Z)$ will represent the energy variables of two different physical energy domains interacting with each other, while $\Omega^{n-p}(\partial Z)$ and $\Omega^{n-q}(\partial Z)$ will denote the boundary variables whose (wedge) product represents the boundary energy flow. For example, in Maxwell's equations (Example 3.1) we will have $n=3$ and $p=q=2$; with $\Omega^{p}(Z)=\Omega^{2}(Z)$, respectively $\Omega^{q}(Z)=\Omega^{2}(Z)$, being the space of electric field inductions, respectively magnetic field inductions, and $\Omega^{n-p}(\partial Z)=\Omega^{1}(\partial Z)$ denoting the electric and magnetic field intensities at the boundary, with product the Poynting vector.

Theorem 3.5. Consider $\mathcal{F}_{p, q}$ and $\mathcal{E}_{p, q}$ given in (50), (52) with $p, q$ satisfying (51), and bilinear form $\ll, \gg$ given by (53). Define the following linear subspace $D$ of $\mathcal{F}_{p, q} \times \mathcal{E}_{p, q}$

$$
\begin{aligned}
D= & \left\{\left(f_{p}, f_{q}, f_{b}, e_{p}, e_{q}, e_{b}\right) \in \mathcal{F}_{p, q} \times \mathcal{E}_{p, q} \mid\right. \\
& {\left[\begin{array}{c}
f_{p} \\
f_{q}
\end{array}\right]=\left[\begin{array}{cc}
0 & (-1)^{r} d \\
d & 0
\end{array}\right]\left[\begin{array}{l}
e_{p} \\
e_{q}
\end{array}\right] } \\
& {\left.\left[\begin{array}{c}
f_{b} \\
e_{b}
\end{array}\right]=\left[\begin{array}{cc}
1 & 0 \\
0 & -(-1)^{n-q}
\end{array}\right]\left[\begin{array}{l}
e_{p \mid \partial Z} \\
e_{q \mid \partial Z}
\end{array}\right]\right\} }
\end{aligned}
$$

where $\left.\right|_{\partial Z}$ denotes restriction to the boundary $\partial Z$, and $r:=p q+1$. Then $D=D^{\perp}$, that is, $D$ is a Dirac structure.

For the proof of this theorem we refer to [37].

Remark 3.6. The spatial compositionality properties of the Stokes-Dirac structure immediately follow from its definition. Indeed, let $Z_{1}, Z_{2}$ be two $n$-dimensional manifolds with boundaries $\partial Z_{1}, \partial Z_{2}$, such that

$$
\begin{aligned}
& \partial Z_{1}=\Gamma \cup \Gamma_{1}, \quad \Gamma \cap \Gamma_{1}=\phi \\
& \partial Z_{2}=\Gamma \cup \Gamma_{2}, \quad \Gamma \cap \Gamma_{2}=\phi
\end{aligned}
$$

for certain $(n-1)$-dimensional manifolds $\Gamma, \Gamma_{1}, \Gamma_{2}$ (that is, $Z_{1}$ and $Z_{2}$ have boundary $\Gamma$ in common). Then the Stokes-Dirac structures $D_{1}, D_{2}$ on $Z_{1}$, respectively $Z_{2}$, compose to the Stokes-Dirac structure on the manifold $Z_{1} \cup Z_{2}$ with boundary $\Gamma_{1} \cup$ $\Gamma_{2}$ if we equate on $\Gamma$ the boundary variables $f_{b}^{1}$ (corresponding to $D_{1}$ ) with $-f_{b}^{2}$ (corresponding to $D_{2}$ ). (Note that a minus sign is inserted in order to ensure that the power flowing into $Z_{1}$ via $\Gamma$ is equal to the power flowing out of $Z_{2}$ via $\Gamma$.) 


\subsection{Poisson brackets associated to Stokes-Dirac structures}

Although Dirac structures strictly generalize Poisson structures we can associate a (pseudo-)Poisson structure to any Dirac structure, as defined in Section 2.1. Indeed, let $D \subset \mathcal{F} \times \mathcal{E}$ be a Dirac structure as given in Definition 3.1. Then we can define a skew-symmetric bilinear form on a subspace of $\mathcal{E}$; basically following [5], [7]. First, define the space of 'admissible efforts'

$$
\mathcal{E}_{\mathrm{adm}}=\{e \in \mathcal{E} \mid \exists f \in \mathcal{F} \text { such that }(f, e) \in D\}
$$

Then we define on $\mathcal{E}_{\text {adm }}$ the bilinear form

$$
\left[e_{1}, e_{2}\right]:=<e_{1} \mid f_{2}>\in L
$$

where $f_{2} \in \mathcal{F}$ is such that $\left(f_{2}, e_{2}\right) \in D$. This bilinear form is well-defined, since for any other $f_{2}^{\prime} \in \mathcal{F}$ such that $\left(f_{2}^{\prime}, e_{2}\right) \in D$ we obtain by linearity $\left(f_{2}-f_{2}^{\prime}, 0\right) \in D$, and hence

$$
0=\ll\left(f_{1}, e_{1}\right),\left(f_{2}-f_{2}^{\prime}, 0\right) \gg=<e_{1}\left|f_{2}>-<e_{1}\right| f_{2}^{\prime}>
$$

Furthermore, $[$,$] is skew-symmetric since for any \left(f_{1}, e_{1}\right),\left(f_{2}, e_{2}\right) \in D$

$$
0=\ll\left(f_{1}, e_{1}\right),\left(f_{2}, e_{2}\right) \gg=<e_{1}\left|f_{2}>+<e_{2}\right| f_{1}>
$$

Now, let us define on $\mathcal{F}$ the set of admissible mappings

$$
\begin{aligned}
K_{\mathrm{adm}}= & \left\{k: \mathcal{F} \rightarrow L \mid \forall a \in \mathcal{F} \exists e(k, a) \in \mathcal{E}_{\text {adm }}\right. \\
& \text { such that for all } \partial a \in \mathcal{F} \\
& k(a+\partial a)=k(a)+<e(k, a) \mid \partial a>+O(\partial a)\}
\end{aligned}
$$

Note that $e(k, a)$ (if it exists) is uniquely defined modulo the following linear subspace of $\mathcal{E}$

$$
\mathcal{E}_{0}=\{e \in \mathcal{E}|<e| f>=0 \quad \text { for all } f \in \mathcal{F}\}
$$

We call $e(k, a)$ (in fact, its equivalence class) the derivative of $k$ at $a$, and we denote it by $\delta k(a)$. We define on $K_{\mathrm{adm}}$ the following bracket

$$
\left\{k_{1}, k_{2}\right\}_{D}(a):=\left[\delta k_{1}(a), \delta k_{2}(a)\right], \quad k_{1}, k_{2} \in K_{\mathrm{adm}}
$$

which is clearly independent from the choice of the representants $\delta k_{1}(a), \delta k_{2}(a)$. By skew-symmetry of [,] it immediately follows that also $\{$,$\} is skew-symmetric.$ The Jacobi-identity for $\{,\}_{D}$, however, is not automatically satisfied, and we call therefore $\{,\}_{D}$ a pseudo-Poisson bracket.

For the Stokes-Dirac structure $D$ of Theorem 3.5, given in equation (55), the bracket takes the following form. The set of admissible functions $K_{\text {adm }}$ consists of those functions

$$
k: \Omega^{p}(z) \times \Omega^{q}(z) \times \Omega^{n-p}(\partial z) \rightarrow \mathbb{R}
$$


whose derivatives

$$
\delta k(a)=\left(\delta_{p} k(a), \delta_{q} k(a), \delta_{b} k(a)\right) \in \Omega^{n-p}(Z) \times \Omega^{n-q}(Z) \times \Omega^{n-q}(\partial Z)
$$

satisfy (cf. the last line of (55)

$$
\delta_{b} k(a)=-\left.(-1)^{n-q} \delta_{q} k(a)\right|_{\partial Z}
$$

Furthermore, the bracket on $K$ adm is given as (leaving out the arguments $a$ )

$$
\begin{array}{r}
\left\{k^{1}, k^{2}\right\}_{D}=\int_{Z}\left[\delta_{p} k^{1} \wedge(-1)^{r} d\left(\delta_{q} k^{2}\right)+\left(\delta_{q} k^{1}\right) \wedge d\left(\delta_{p} k^{2}\right)\right] \\
-\int_{\partial Z}(-1)^{n-q}\left(\delta_{q} k^{1}\right) \wedge\left(\delta_{p} k^{2}\right)
\end{array}
$$

It follows from the general considerations above that this bracket is skew-symmetric. (This can be also directly checked using Stokes' theorem.) Furthermore, in this case it is straightforward to check that $\{,\}_{D}$ also satisfies the Jacobi-identity

$$
\left.\left.\left\{\left\{k^{1}, k^{2}\right\}_{D}, k^{3}\right\}_{D}+\left\{\left\{k^{2}, k^{3}\right\}_{D}, k^{1}\right\}\right\}+\left\{k^{3}, k^{1}\right\}_{D}, k^{2}\right\}_{D}=0
$$

for all $k^{i} \in K$ adm .

\section{Hamiltonian formulation of distributed-parameter sys- tems with boundary energy flow}

\subsection{Boundary port-Hamiltonian systems}

The definition of a distributed-parameter Hamiltonian system with respect to a Stokes-Dirac structure can now be stated as follows. Let $Z$ be an $n$-dimensional manifold with boundary $\partial Z$, and let $D$ be a Stokes-Dirac structure as in Subsection 2.2. Consider furthermore a Hamiltonian density (energy per volume element)

$$
\mathcal{H}: \Omega^{p}(Z) \times \Omega^{q}(Z) \times Z \rightarrow \Omega^{n}(Z)
$$

resulting in the total energy

$$
H:=\int_{Z} \mathcal{H} \in \mathbb{R}
$$

Let $\alpha_{p}, \partial \alpha_{p} \in \Omega^{p}(Z), \alpha_{q}, \partial \alpha_{q} \in \Omega^{q}(Z)$. Then (with $z \in Z$ )

$$
\begin{array}{r}
H\left(\alpha_{p}+\partial \alpha_{p}, \alpha_{q}+\partial \alpha_{q}\right)= \\
\int_{Z} \mathcal{H}\left(\alpha_{p}+\partial \alpha_{p}, \alpha_{q}+\partial \alpha_{q}, z\right)= \\
\int_{Z} \mathcal{H}\left(\alpha_{p}, \alpha_{q}, z\right)+\int_{Z}\left[\delta_{p} H \wedge \partial \alpha_{p}+\delta_{q} H \wedge \partial \alpha_{q}\right] \\
+ \text { higher order terms in } \partial \alpha_{p}, \partial \alpha_{q}
\end{array}
$$


for certain uniquely defined differential forms

$$
\begin{aligned}
& \delta_{p} H \in \Omega^{n-p}(Z) \\
& \delta_{q} H \in \Omega^{n-q}(Z)
\end{aligned}
$$

This means that $\left(\delta_{p} H, \delta_{q} H\right) \in \Omega^{n-p}(Z) \times \Omega^{n-q}(Z)$ can be regarded as the variational derivative of $H$ at $\left(\alpha_{p}, \alpha_{q}\right) \in \Omega^{p}(Z) \times \Omega^{q}(Z)$.

Now consider a time-function

$$
\left(\alpha_{p}(t), \alpha_{q}(t)\right) \in \Omega^{p}(Z) \times \Omega^{q}(Z), \quad t \in \mathbb{R},
$$

and the Hamiltonian $H\left(\alpha_{p}(t), \alpha_{q}(t)\right)$ evaluated along this trajectory. It follows that at any time $t$

$$
\frac{d H}{d t}=\int_{Z}\left[\delta_{p} H \wedge \frac{\partial \alpha_{p}}{\partial t}+\delta_{q} H \wedge \frac{\partial \alpha_{q}}{\partial t}\right]
$$

The differential forms $\frac{\partial \alpha_{p}}{\partial t}, \frac{\partial \alpha_{q}}{\partial t}$ represent the generalized velocities of the energy variables $\alpha_{p}, \alpha_{q}$. They are connected to the Stokes-Dirac structure $D$ by setting

$$
\begin{aligned}
& f_{p}=-\frac{\partial \alpha_{p}}{\partial t} \\
& f_{q}=-\frac{\partial \alpha_{q}}{\partial t}
\end{aligned}
$$

(again the minus sign is included to have a consistent energy flow description). Since the right-hand side of (74) is the rate of increase of the stored energy $H$, we set

$$
\begin{aligned}
& e_{p}=\delta_{p} H \\
& e_{q}=\delta_{q} H
\end{aligned}
$$

Now we come to the general Hamiltonian description of a distributed-parameter system with boundary energy flow. In order to emphasize that the boundary variables are regarded as interconnection variables, which can be interconnected to other systems and whose product represents power, we call these models port-Hamiltonian systems. (This terminology comes from network modelling, see e.g. [23], [35], [34].)

Definition 4.1. The boundary port-Hamiltonian system with $n$-dimensional manifold of spatial variables $Z$, state space $\Omega^{p}(Z) \times \Omega^{q}(Z)$ (with $p+q=n+1$ ), StokesDirac structure $D$ given by (55), and Hamiltonian $H$, is given as (with $r=p q+1$ )

$$
\begin{aligned}
{\left[\begin{array}{c}
-\frac{\partial \alpha_{p}}{\partial t} \\
-\frac{\partial \alpha_{q}}{\partial t}
\end{array}\right] } & =\left[\begin{array}{cc}
0 & (-1)^{r} d \\
d & 0
\end{array}\right]\left[\begin{array}{l}
\delta_{p} H \\
\delta_{q} H
\end{array}\right] \\
{\left[\begin{array}{c}
f_{b} \\
e_{b}
\end{array}\right] } & =\left[\begin{array}{cc}
1 & 0 \\
0 & -(-1)^{n-q}
\end{array}\right]\left[\begin{array}{l}
\left.\delta_{p} H\right|_{\partial Z} \\
\left.\delta_{q} H\right|_{\partial Z}
\end{array}\right]
\end{aligned}
$$


By the power-conserving property (47) of any Dirac structure it immediately follows that for any $\left(f_{p}, f_{q}, f_{b}, e_{p}, e_{q}, e_{b}\right)$ in the Stokes-Dirac structure $D$

$$
\int_{Z}\left[e_{p} \wedge f_{p}+e_{q} \wedge f_{q}\right]+\int_{\partial Z} e_{b} \wedge f_{b}=0
$$

Hence by substitution of (75), (76) and using (74) we obtain

Proposition 4.2. Consider the distributed parameter port-Hamiltonian system (77). Then

$$
\frac{d H}{d t}=\int_{\partial Z} e_{b} \wedge f_{b}
$$

expressing that the increase in energy on the domain $Z$ is equal to the power supplied to the system through the boundary $\partial Z$.

The system (77) can be called a (nonlinear) boundary control system in the sense of e.g. [9]. Indeed, we could interpret $f_{b}$ as the boundary control inputs to the system, and $e_{b}$ as the measured outputs (or the other way around). In Section 6 we shall further elaborate on this point of view.

\subsection{Boundary port-Hamiltonian systems with distributed ports and dissipation}

Energy exchange through the boundary is not the only way a distributed-parameter system may interact with its environment. An example of this is provided by Maxwell's equations (Example 5.1), where interaction may also take place via the current density $J$, which directly affects the electric charge distribution in the domain $Z$. In order to cope with this situation we augment the spaces $\mathcal{F}_{p, q}, \mathcal{E}_{p, q}$ as defined in (50), (52) to

$$
\begin{aligned}
\mathcal{F}_{q, p}^{a} & :=\mathcal{F}_{p, q} \times \Omega^{d}(S) \\
\mathcal{E}_{q, p}^{a} & :=\mathcal{E}_{p, q} \times \Omega^{n-d}(S)
\end{aligned}
$$

for some $m$-dimensional manifold $S$ and some $d \in\{0,1, \cdots, m\}$, with $f^{d} \in \Omega^{d}(S)$ denoting the externally supplied distributed control flow, and $e^{d} \in \Omega^{n-d}(S)$ the conjugate distributed quantity, corresponding to an energy exchange

$$
\int_{S} e^{d} \wedge f^{d}
$$


The Stokes-Dirac structure (55) is now extended to

$$
\begin{aligned}
{\left[\begin{array}{l}
f_{p} \\
f_{q}
\end{array}\right] } & =\left[\begin{array}{cc}
0 & (-1)^{r} d \\
d & 0
\end{array}\right]\left[\begin{array}{l}
e_{p} \\
e_{q}
\end{array}\right]+G\left(f_{d}\right) \\
{\left[\begin{array}{c}
f_{b} \\
e_{b}
\end{array}\right] } & =\left[\begin{array}{cc}
1 & 0 \\
0 & -(-1)^{n-q}
\end{array}\right]\left[\begin{array}{l}
e_{p \mid \partial Z} \\
e_{q \mid \partial Z}
\end{array}\right] \\
e_{d} & =-G^{*}\left[\begin{array}{l}
e_{p} \\
e_{q}
\end{array}\right]
\end{aligned}
$$

with $G$ denoting a linear map

$$
G=\left(\begin{array}{c}
G_{p} \\
G_{q}
\end{array}\right): \Omega^{d}(S) \rightarrow \Omega^{p}(Z) \times \Omega^{q}(Z)
$$

with dual map

$$
G^{*}=\left(G_{p}^{*}, G_{q}^{*}\right): \Omega^{n-p}(Z) \times \Omega^{n-q}(Z) \rightarrow \Omega^{n-d}(S)
$$

satisfying

$$
\int_{Z}\left[e_{p} \wedge G_{p}\left(f_{d}\right)+e_{q} \wedge G_{q}\left(f_{d}\right)\right]=\int_{S}\left[G_{p}^{*}\left(e_{p}\right)+G_{q}^{*}\left(e_{q}\right)\right] \wedge f_{d}
$$

for all $e_{p} \in \Omega^{n-p}(Z), e_{q} \in \Omega^{n-q}(Z), f_{d} \in \Omega^{d}(S)$.

The following proposition can be easily checked.

Proposition 4.3. Equations (82) determine a Dirac structure $D^{a} \subset \mathcal{F}_{p, q}^{a} \times \mathcal{E}_{p, q}^{a}$ with respect to the augmented bilinear form on $\mathcal{F}_{p, q}^{a} \times \mathcal{E}_{p, q}^{a}$ which is obtained by adding to the bilinear form $(53)$ on $\mathcal{F}_{p, q} \times \mathcal{E}_{p, q}$ the term

$$
\int_{S}\left[e_{d}^{1} \wedge f_{d}^{2}+e_{d}^{2} \wedge f_{d}^{1}\right]
$$

By making now the substitutions $(75),(76)$ into $D^{a}$ given by (82) we obtain a port-Hamiltonian system with external variables $\left(f_{b}, f_{d}, e_{b}, e_{d}\right)$, with $f_{b}, e_{b}$ the boundary external variables and $f_{d}, e_{d}$ the distributed external variables. Furthermore, the energy balance (79) extends to

$$
\frac{d H}{d t}=\int_{\partial Z} e_{b} \wedge f_{b}+\int_{S} e_{d} \wedge f_{d}
$$

with the first term on the right-hand side denoting the power flow through the boundary, and the second term denoting the distributed power flow.

Finally, energy dissipation can be incorporated in the framework of distributedparameter port-Hamiltonian systems by terminating some of the ports (boundary or distributed) with a resistive relation. For example, for distributed dissipation, let $R: \Omega^{n-d}(S) \rightarrow \Omega^{d}(S)$ be a map satisfying

$$
\int_{S} e_{d} \wedge R\left(e_{d}\right) \geq 0, \quad \forall e_{d} \in \Omega^{n-d}(S)
$$


Then by adding the relation

$$
f_{d}=-R\left(e_{d}\right)
$$

to the port-Hamiltonian system defined with respect to the Dirac structure $D^{a}$, we obtain a port-Hamiltonian system with dissipation, satisfying the energy inequality

$$
\frac{d H}{d t}=\int_{\partial Z} e_{b} \wedge f_{b}-\int_{S} e_{d} \wedge R\left(e_{d}\right) \leq \int_{\partial Z} e_{b} \wedge f_{b}
$$

\section{$5 \quad$ Examples}

In this section we show how the framework of distributed-parameter port-Hamiltonian systems admits the representation of Maxwell's equations, the telegraph equations of an ideal transmission line, the vibrating string, and the Euler equations of an ideal isentropic fluid.

\subsection{Maxwell's equations}

We closely follow the formulation of Maxwell's equations in terms of differential forms as presented in [12], and show how this directly leads to the formulation as a distributed-parameter port-Hamiltonian system.

Let $Z \subset \mathbb{R}^{3}$ be a 3 -dimensional manifold with boundary $\partial Z$, defining the spatial domain, and consider the electromagnetic field in $Z$. The energy variables are the electric field induction 2-form $\alpha_{p}=\mathcal{D} \in \Omega^{2}(Z)$ :

$$
\mathcal{D}=\frac{1}{2} D_{i j}(t, z) d z^{i} \wedge d z^{j}
$$

and the magnetic field induction 2-form $\alpha_{q}=\mathcal{B} \in \Omega^{2}(Z)$ :

$$
\mathcal{B}=\frac{1}{2} B_{i j}(t, z) d z^{i} \wedge d z^{j}
$$

The corresponding Stokes-Dirac structure $(n=3, p=2, q=2)$ is given as (cf. (55))

$$
\left[\begin{array}{l}
f_{p} \\
f_{q}
\end{array}\right]=\left[\begin{array}{cc}
0 & -d \\
d & 0
\end{array}\right]\left[\begin{array}{l}
e_{p} \\
e_{q}
\end{array}\right],\left[\begin{array}{l}
f_{b} \\
e_{b}
\end{array}\right]=\left[\begin{array}{ll}
1 & 0 \\
0 & 1
\end{array}\right]\left[\begin{array}{l}
e_{p \mid \partial Z} \\
e_{q \mid \partial Z}
\end{array}\right]
$$

Usually in this case one does not start with the definition of the total energy (Hamiltonian) $H$, but instead with the co-energy variables $\delta_{p} H, \delta_{q} H$, given, respectively, as the electric field intensity $\mathcal{E} \in \Omega^{1}(Z)$ :

$$
\mathcal{E}=E_{i}(t, z) d z^{i}
$$

and the magnetic field intensity $\mathcal{H} \in \Omega^{1}(Z)$ :

$$
\mathcal{H}=H_{i}(t, z) d z^{i}
$$


They are related to the energy variables through the constitutive relations of the medium (or material equations)

$$
\begin{aligned}
* \mathcal{D} & =\epsilon \mathcal{E} \\
* \mathcal{B} & =\mu H
\end{aligned}
$$

with the scalar functions $\epsilon(t, z)$ and $\mu(t, z)$ denoting the electric permittivity, respectively magnetic permeability, and $*$ denoting the Hodge star operator (corresponding to a Riemannian metric on $Z$ ), converting 2-forms into 1-forms. Then one defines the Hamiltonian $H$ as

$$
H=\int_{Z} \frac{1}{2}(\mathcal{E} \wedge \mathcal{D}+\mathcal{H} \wedge \mathcal{B})
$$

and one immediately verifies that $\delta_{p} H=\mathcal{E}, \delta_{q} H=\mathcal{H}$.

Nevertheless there are other cases (corresponding to a nonlinear theory of the electromagnetic field, such as the Born-Infeld theory, see e.g. [12]) where one starts with a more general Hamiltonian $H=\int_{Z} h$, with the energy density $h(\mathcal{D}, B)$ being a more general expression than $\frac{1}{2}\left(\epsilon^{-1} * \mathcal{D} \wedge \mathcal{D}+\mu^{-1} * \mathcal{B} \wedge \mathcal{B}\right)$.

Assuming that there is no current in the medium Maxwell's equations can now be written as (see [12])

$$
\begin{aligned}
& \frac{\partial \mathcal{D}}{\partial t}=d \mathcal{H} \\
& \frac{\partial \mathcal{B}}{\partial t}=-d \mathcal{E}
\end{aligned}
$$

Explicitly taking into account the behavior at the boundary, Maxwell's equations on a domain $Z \subset \mathbb{R}^{3}$ are then represented as the port-Hamiltonian system with respect to the Stokes-Dirac structure given by (93), as

$$
\begin{aligned}
{\left[\begin{array}{c}
-\frac{\partial \mathcal{D}}{\partial t} \\
-\frac{\partial B}{\partial t}
\end{array}\right] } & =\left[\begin{array}{cc}
0 & -d \\
d & 0
\end{array}\right]\left[\begin{array}{l}
\delta_{D} H \\
\delta_{B} H
\end{array}\right] \\
{\left[\begin{array}{c}
f_{b} \\
e_{b}
\end{array}\right] } & =\left[\begin{array}{l}
\left.\delta_{D} H\right|_{\partial Z} \\
\left.\delta_{B} H\right|_{\partial Z}
\end{array}\right]
\end{aligned}
$$

Note that the first line of (98) is nothing else than (the differential version of) Ampère's law, while the second line of (98) is Faraday's law. Hence the StokesDirac structure in (98), (99) expresses the basic physical laws connecting $\mathcal{D}, \mathcal{B}, \mathcal{H}$ and $\mathcal{E}$.

The energy-balance (79) in the case of Maxwell's equations takes the form

$$
\frac{d H}{d t}=\int_{\partial Z} \delta_{B} H \wedge \delta_{D} H=\int_{\partial Z} \mathcal{H} \wedge \mathcal{E}=-\int_{\partial Z} \mathcal{E} \wedge \mathcal{H}
$$

with $\mathcal{E} \wedge \mathcal{H}$ a 2 -form corresponding to the Poynting vector (see [12]).

In the case of a non-zero current density we have to modify the first matrix equation of (99) to

$$
\left[\begin{array}{l}
-\frac{\partial D}{\partial t} \\
-\frac{\partial B}{\partial t}
\end{array}\right]=\left[\begin{array}{cc}
0 & -d \\
d & 0
\end{array}\right]\left[\begin{array}{c}
\delta_{D} H \\
\delta_{B} H
\end{array}\right]+\left[\begin{array}{l}
I \\
0
\end{array}\right] J
$$


with $I$ denoting the identity operator from $J \in \Omega^{2}(Z)$ to $\Omega^{2}(Z)$. (Thus, in the notation of (83), $f_{d}=J, S=Z$, and $\Omega^{d}(S)=\Omega^{2}(Z)$.) Furthermore, we add the equation

$$
e_{d}=-\left[\begin{array}{ll}
I & 0
\end{array}\right]\left[\begin{array}{l}
\delta_{D} H \\
\delta_{B} H
\end{array}\right]=-\mathcal{E}
$$

yielding the augmented energy balance

$$
\frac{d H}{d t}=-\int_{\partial Z} \mathcal{E} \wedge \mathcal{H}-\int_{Z} \mathcal{E} \wedge J
$$

which is known as Poynting's theorem.

Finally, in order to incorporate energy dissipation we write $J=J_{d}+\bar{J}$, and we impose Ohm's law

$$
* J_{d}=\sigma \mathcal{E}
$$

with $\sigma(t, z)$ the specific conductivity of the medium.

\subsection{Telegraph equations}

Consider an ideal lossless transmission line with $Z=[0,1] \subset \mathbb{R}$. The energy variables are the charge density 1-form $Q=Q(t, z) d z \in \Omega^{1}([0,1])$, and the flux density 1-form $\varphi=\varphi(t, z) d z \in \Omega^{1}([0,1])$; thus $p=q=n=1$. The total energy stored at time $t$ in the transmission line is given as

$$
H(Q, \varphi)=\int_{0}^{1} \frac{1}{2}\left(\frac{Q^{2}(t, z)}{C(z)}+\frac{\varphi^{2}(t, z)}{L(z)}\right) d z
$$

with co-energy variables

$$
\begin{aligned}
& \delta_{Q} H=\frac{Q(t, z)}{C(z)}=V(t, z) \quad \text { (voltage) } \\
& \delta_{Q} H=\frac{\varphi(t, z)}{L(z)}=I(t, z) \quad \text { (current) }
\end{aligned}
$$

where $C(z), L(z)$ are respectively the distributed capacitance and distributed inductance of the line.

The resulting port-Hamiltonian system is given by the telegraph equations

$$
\begin{aligned}
& \frac{\partial Q}{\partial t}=-\frac{\partial I}{\partial z} \\
& \frac{\partial \varphi}{\partial t}=-\frac{\partial V}{\partial z}
\end{aligned}
$$

together with the boundary variables

$$
\begin{aligned}
& f_{b}^{0}(t)=V(t, 0), \quad f_{b}^{1}(t)=V(t, 1) \\
& e_{b}^{0}(t)=-I(t, 0), \quad e_{b}^{1}(t)=-I(t, 1)
\end{aligned}
$$

The resulting energy-balance is

$$
\frac{d H}{d t}=\int_{\partial([0,1])} e_{b} f_{b}=-I(t, 1) V(t, 1)+I(t, 0) V(t, 0)
$$

in accordance with (79). 


\subsection{Vibrating string}

Consider an elastic string subject to traction forces at its ends. The spatial variable $z$ belongs to the interval $Z=[0,1] \subset \mathbb{R}$. Let us denote by $u(t, z)$ the displacement of the string. The elastic potential energy is a function of the strain given by the 1 -form

$$
\alpha_{q}(t)=\epsilon(t, z) d z=\frac{\partial u}{\partial z}(t, z) d z
$$

The associated co-energy variable is the stress given by the 0 -form

$$
\sigma=T * \alpha_{q}
$$

with $T$ the elasticity modulus and $*$ the Hodge star operator. Hence the potential energy is the quadratic function

$$
U\left(\alpha_{q}\right)=\int_{0}^{1} \sigma \alpha_{q}=\int_{0}^{1} T * \alpha_{q} \wedge \alpha_{q}=\int_{0}^{1} T\left(\frac{\partial u}{\partial z}\right)^{2} d z
$$

and $\sigma=\delta_{q} U$.

The kinetic energy $K$ is a function of the kinetic momentum defined as the 1 -form

$$
\alpha_{p}(t)=p(t, z) d z
$$

given by the quadratic function

$$
K\left(\alpha_{p}\right)=\int_{0}^{1} \frac{p^{2}}{\mu} d z
$$

The associated co-energy variable is the velocity given by the 0 -form

$$
v=\frac{1}{\mu} * \alpha_{p}=\delta_{p} K
$$

In this case the Dirac structure is the Stokes-Dirac structure for $n=p=q=1$, with an opposite sign convention leading to the equations (with $H:=U+K$ )

$$
\begin{aligned}
{\left[\begin{array}{c}
-\frac{\partial \alpha_{p}}{\partial t} \\
-\frac{\partial \alpha_{q}}{\partial t}
\end{array}\right] } & =\left[\begin{array}{cc}
0 & -d \\
-d & 0
\end{array}\right]\left[\begin{array}{c}
\delta_{p} H \\
\delta_{q} H
\end{array}\right] \\
{\left[\begin{array}{c}
f_{b} \\
e_{b}
\end{array}\right] } & =\left[\begin{array}{ll}
1 & 0 \\
0 & 1
\end{array}\right]\left[\begin{array}{l}
\left.\delta_{p} H\right|_{\partial Z} \\
\left.\delta_{q} H\right|_{\partial Z}
\end{array}\right]
\end{aligned}
$$

or, in more down-to-earth notation

$$
\begin{aligned}
\frac{\partial p}{\partial t} & =\frac{\partial \sigma}{\partial z}=\frac{\partial}{\partial z}(T \epsilon) \\
\frac{\partial \epsilon}{\partial t} & =\frac{\partial v}{\partial z}=\frac{\partial}{\partial z}\left(\frac{1}{\mu} p\right) \\
f_{b} & =\left.v\right|_{\{0,1\}} \\
e_{b} & =\left.\sigma\right|_{\{0,1\}}
\end{aligned}
$$


with boundary variables the velocity and stress at the ends of the string. Of course, by substituting $\epsilon=\frac{\partial u}{\partial z}$ into the 2 nd equation of (117) one obtains $\frac{\partial}{\partial z}\left(\frac{\partial u}{\partial t}-\frac{p}{\mu}\right)=0$, implying that

$$
p=\mu \frac{\partial u}{\partial t}+\mu f(t)
$$

for some function $f$, which may be set to zero. Substitution of (118) into the first equation of (117) then yields the wave equation

$$
\mu \frac{\partial^{2} u}{\partial t^{2}}=\frac{\partial}{\partial z}\left(T \frac{\partial u}{\partial z}\right)
$$

\section{Extension of port-Hamiltonian systems defined on Stokes- Dirac structures}

\subsection{Burger's equations}

Consider the inviscid Burger's equation as discussed in Section 2.1. Consider $Z$ to be a bounded interval of $\mathbb{R}$, then Burger's inviscid equations are:

$$
\frac{\partial u}{\partial t}+\frac{\partial}{\partial x}\left(\frac{u^{2}}{2}\right)=0
$$

which is a scalar convex conservation equation.

It may be formulated as a boundary control system as follows:

$$
\begin{aligned}
\frac{\partial u}{\partial t} & =-\frac{\partial}{\partial x}\left(\delta_{u} H\right) \\
w_{b} & =\left.\delta_{u} H\right|_{\partial Z}
\end{aligned}
$$

where $\delta_{u} H$ denotes the variational derivative of the Hamiltonian functional $H(u)=$ $\frac{1}{6} u^{3}$. Defining the power-conjugated variables to be $f=\frac{\partial u}{\partial t}, e=\delta_{u} H$ and on the boundary $w_{b}$, one may define an infinite-dimensional Dirac structure which is different from the the Stokes-Dirac structure. With regard to this Dirac structure the inviscid Burger's equation is represented as a distributed port-Hamiltonian system. For details we refer to [15].

\subsection{Ideal isentropic fluid}

Consider an ideal compressible isentropic fluid in three dimensions, described in Eulerian representation by the standard Euler equations

$$
\begin{aligned}
& \frac{\partial \rho}{\partial t}=-\nabla \cdot(\rho v) \\
& \frac{\partial v}{\partial t}=-v \cdot \nabla v-\frac{1}{\rho} \nabla p
\end{aligned}
$$

with $\rho(z, t) \in \mathbb{R}$ the mass density at the spatial position $z \in \mathbb{R}^{3}$ at time $t, v(z, t) \in \mathbb{R}^{3}$ the (Eulerian) velocity of the fluid at spatial position $z$ and time $t$, and $p(z, t)$ the 
pressure function, derivable from an internal energy function $U(\rho)$ as

$$
p(z, t)=\rho^{2}(z, t) \frac{\partial U}{\partial \rho}(\rho(z, t))
$$

Much innovative work has been done regarding the Hamiltonian formulation of (125) and more general cases; we refer in particular to [24, 17, 18, 25, 14]. However, in these treatments only closed fluid dynamical systems are being considered with no energy exchange through the boundary of the spatial domain. As a result, a formulation in terms of Poisson structures can be given, while as argued before, the general inclusion of boundary variables necessitates the use of Dirac structures.

The formulation of (120) as a port-Hamiltonian system is given as follows. Let $\mathcal{D} \subset \mathbb{R}^{3}$ be a given domain, filled with the fluid. We assume the existence of a Riemannian metric $<,>$ on $\mathcal{D}$; usually the standard Euclidean metric on $\mathbb{R}^{3}$. Let $Z \subset \mathcal{D}$ be any 3-dimensional manifold with boundary $\partial Z$.

We identify the mass-density $\rho$ with a 3 -form on $Z$ (see e.g. $[17,18]$ ), that is, with an element of $\Omega^{3}(Z)$. Furthermore, we identify the Eulerian vector field $v$ with a 1 -form on $Z$, that is, with an element of $\Omega^{1}(Z)$. (By the existence of the Riemannian metric on $Z$ we can, by "index raising" or "index lowering", identify vector fields with 1-forms and vice versa.) The precise motivation for this choice of variables will become clear later on. As a result we consider as the carrier spaces for the port-Hamiltonian formulation of (120) the linear spaces $\mathcal{F}_{p, q}$ and $\mathcal{E}_{p, q}$ for $n=3, p=3, q=1$; that is

$$
\mathcal{F}_{p, q}=\Omega^{3}(Z) \times \Omega^{1}(Z) \times \Omega^{0}(\partial Z)
$$

and

$$
\mathcal{E}_{p, q}=\Omega^{0}(Z) \times \Omega^{2}(Z) \times \Omega^{2}(\partial Z)
$$

Since $p+q=n+1$ we can define the corresponding Stokes-Dirac structure $D$ given by (55) on $\mathcal{F}_{p, q} \times \mathcal{E}_{p, q}$. However, as will become clear later on, due to 3-dimensional convection we need to modify this Stokes-Dirac structure with an additional term into the following modified Stokes-Dirac structure

$$
\begin{aligned}
D^{m}:= & \left\{\left(f_{p}, f_{v}, f_{b}, e_{\rho}, e_{v}, e_{b}\right) \in\right. \\
& \Omega^{3}(Z) \times \Omega^{1}(Z) \times \Omega^{0}(\partial Z) \times \Omega^{0}(Z) \times \Omega^{2}(Z) \times \Omega^{2}(\partial Z) \\
& {\left[\begin{array}{c}
f_{\rho} \\
f_{v}
\end{array}\right]=\left[\begin{array}{c}
d e_{v} \\
d e_{\rho}+\frac{1}{* \rho} *\left((* d v) \wedge\left(* e_{v}\right)\right)
\end{array}\right] } \\
& {\left.\left[\begin{array}{c}
f_{b} \\
e_{b}
\end{array}\right]=\left[\begin{array}{c}
e_{\rho \mid \partial Z} \\
-e_{v \mid \partial Z}
\end{array}\right]\right\} }
\end{aligned}
$$

where $*$ denotes the Hodge star operator (corresponding to the Riemannian metric on $Z$ ), converting $k$-forms on $Z$ to $(3-k)$-forms. A fundamental difference of the modified Stokes-Dirac structure $D^{m}$ with respect to the standard Stokes-Dirac 
structure $D$ is that $D^{m}$ explicitly depends on the energy variables $\rho$ and $v$ (via the terms $* \rho$ and $d v$ in the additional term $\frac{1}{* \rho} *\left((* d v) \wedge\left(* e_{v}\right)\right)$.

Completely similar to the proof of Theorem 5 it is shown that $\left(D^{m}(\rho, v)\right)^{\perp}=$ $D^{m}(\rho, v)$ for all $\rho, v$; the crucial additional observation is that the expression

$$
e_{v}^{2} \wedge *\left((* d v) \wedge\left(* e_{v}^{1}\right)\right)
$$

is skew-symmetric in $e_{v}^{1}, e_{v}^{2} \in \Omega^{2}(Z)$.

Remark 6.1. In the standard Euclidean metric, identifying via the Hodge star operator 2 -forms $\beta_{i}$ with 1 -forms, and representing 1-forms as vectors, we have in vector calculus notation the equality

$$
\beta_{2} \wedge *\left(\alpha \wedge * \beta_{1}\right)=\alpha \cdot\left(\beta_{1} \times \beta_{2}\right)
$$

for all 2 -forms $\beta_{1}, \beta_{2}$ and 1 -forms $\alpha$. This shows clearly the skew-symmetry of (125).

The Eulerian equations (120) for an ideal isentropic fluid are obtained in the port-Hamiltonian representation by considering the Hamiltonian

$$
H(\rho, v):=\int_{Z}\left[\frac{1}{2}<v^{\sharp}, v^{\sharp}>\rho+U(* \rho) \rho\right]
$$

with $v^{\sharp}$ the vector field corresponding to the 1-form $v$ ("index lowering"), and $U(* \rho)$ the potential energy. Indeed, by making the substitutions (75), (76) in $D^{m}$, and noting that

$$
\operatorname{grad} H=\left(\delta_{\rho} H, \delta_{v} H\right)=\left(\frac{1}{2}<v^{\sharp}, v^{\sharp}>+\frac{\partial}{\partial \tilde{\rho}}(\tilde{\rho} U(\tilde{\rho})), \quad i_{v^{\sharp}} \rho\right)
$$

with $\tilde{\rho}:=* \rho$, the port-Hamiltonian system takes the form

$$
\begin{aligned}
-\frac{\partial \rho}{\partial t} & =d\left(i_{v^{\sharp}} \rho\right) \\
-\frac{\partial v}{\partial t} & =d\left(\frac{1}{2}<v^{\sharp}, v^{\sharp}>+w(* \rho)\right)+\frac{1}{* \rho}\left((* d v) \wedge\left(* i_{\left.v^{\sharp} \rho\right)}\right)\right. \\
f_{b} & =\left[\frac{1}{2}<v^{\sharp}, v^{\sharp}>+w(* \rho)\right]_{\mid \partial Z} \\
e_{b} & =-i_{v^{\sharp}} \rho_{\mid \partial Z}
\end{aligned}
$$

with

$$
w(\tilde{\rho}):=\frac{\partial}{\partial \tilde{\rho}}(\tilde{\rho} U(\tilde{\rho}))
$$

the enthalpy. The expression $\left.\delta_{\rho} H=\frac{1}{2}<v^{\sharp}, v^{\sharp}\right\rangle+w(\tilde{\rho})$ is known as the Bernoulli function.

The first two equations of (129) can be seen to represent the Eulerian equations (120). The first equation corresponds to the basic law of mass-balance

$$
\frac{d}{d t} \int_{\varphi t(V)} \rho=0
$$


where $V$ denotes an arbitrary volume in $Z$, and $\varphi_{t}$ is the flow of the fluid (transforming the material volume $V$ at $t=0$ to the volume $\varphi_{t}(V)$ at time $t$ ). Indeed, (131) for any $V$ is equivalent to

$$
\frac{\partial \rho}{\partial t}+L_{v^{\sharp}} \rho=0
$$

Since by Cartan's magical formula $L_{v^{\sharp}} \rho=d\left(i_{v^{\sharp}} \rho\right)+i_{v^{\sharp}} d \rho=d\left(i_{v^{\sharp}} \rho\right)$ (since $d \rho=0$ ) this yields the first line of (129). It also makes clear the interpretation of $\rho$ as a 3-form on $Z$.

For the identification of the second equation of (129) with the second equation of $(125)$ we note the following (see [36] for further details). Interpret $\nabla \cdot$ in $(120)$ as the covariant derivative corresponding to the assumed Riemannian metric $<$, > on $Z$. For a vector field $u$ on $Z$, let $u^{b}$ denote the corresponding 1 -form $u^{b}:=i_{u}<,>$ ("index raising"). The covariant derivative $\nabla$ is related to the Lie derivative by the following formula (see for a proof [14], p. 202)

$$
L_{u} u^{b}=\left(\nabla_{u} u\right)^{b}+\frac{1}{2} d<u, u>
$$

Since by Cartan's magical formula $L_{u} u^{b}=i_{u} d u^{b}+d\left(i_{u} u^{b}\right)=i_{u} d u^{b}+d<u, u>$, (133) can be also written as

$$
\left(\nabla_{u} u\right)^{b}=i_{u} d u^{b}+\frac{1}{2} d<u, u>
$$

(This is the coordinate-free analog of the well-known vector calculus formula $u \cdot \nabla u=$ curl $u \times u+\frac{1}{2} \nabla|u|^{2}$.) Furthermore we have the identity

$$
i_{v^{\sharp}} d v=\frac{1}{* \rho} *\left((* d v) \wedge\left(* i_{v^{\sharp}} \rho\right)\right)
$$

Finally, we have the following well-known relation between enthalpy and pressure (obtained from (126) and (130))

$$
\frac{1}{\tilde{\rho}} d p=d(w(\tilde{\rho})) .
$$

Hence by (134) (with $\left.u=v^{\sharp}\right)$, (110) and (136), we may rewrite the 2nd equation of (129) as

$$
-\frac{\partial v}{\partial t}=\left(\nabla_{v^{\sharp}} v^{\sharp}\right)^{b}+\frac{1}{* \rho} d p
$$

which is the coordinate-free formulation of the 2nd equation of (120).

The boundary variables $f_{b}$ and $e_{b}$ given in (129) are respectively the stagnation pressure at the boundary divided by $\rho$, and the (incoming) mass flow through the boundary. The energy-balance (79) can be written out as

$$
\begin{aligned}
\frac{d H}{d t} & =\int_{\partial Z} e_{b} \wedge f_{b}=-\int_{\partial Z} i_{v^{\sharp}} \rho \wedge\left[\frac{1}{2}<v^{\sharp}, v^{\sharp}>+w(* \rho)\right] \\
& =-\int_{\partial Z} i_{v^{\sharp}}\left[\frac{1}{2}<v^{\sharp}, v^{\sharp}>\rho+w(* \rho) \rho\right] \\
& =-\int_{\partial Z} i_{v^{\sharp}}\left[\frac{1}{2}<v^{\sharp}, v^{\sharp}>\rho+U(* \rho) \rho\right]-\int_{\partial Z} i_{v^{\sharp}}(* p)
\end{aligned}
$$


where for the last equality we have used the relation (following from (121), (130))

$$
w(* \rho) \rho=U(* \rho) \rho+* p
$$

The first term in the last line of (138) corresponds to the convected energy through the boundary $\partial Z$, while the second term is (minus) the external work (static pressure times velocity).

Usually, the second line of the Euler equations (120) (or equivalently equation (137)) is obtained from the basic conservation law of momentum-balance together with the first line of (120). Alternatively, emphasizing the interpretation of $v$ as a 1 -form, we may obtain it from Kelvin's circulation theorem

$$
\frac{d}{d t} \int_{\varphi_{t}(C)} v=0
$$

where $C$ denotes any closed contour. Indeed, (140) for any closed $C$ is equivalent to the 1-form $\frac{\partial v}{\partial t}+L_{v^{\sharp}} v$ being closed. By (133) this is equivalent to requiring

$$
\frac{\partial v}{\partial t}+\left(\nabla_{v^{\sharp}} v^{\sharp}\right)^{b}
$$

to be closed, that is

$$
\frac{\partial v}{\partial t}+\left(\nabla_{v^{\sharp}} v^{\sharp}\right)^{b}=-d k
$$

for some (possibly locally defined) $k: Z \rightarrow \mathbb{R}$. Now additionally requiring that this function $k$ depends on $z$ through $\rho$, that is

$$
k(z)=w(\rho(z))
$$

for some function $w$, we recover (137) with $\frac{1}{* \rho} d p$ replaced by $d w$ (the differential of the enthalpy).

Remark 6.2. In the case of a one- or two-dimensional fluid flow the extra term in the Dirac structure $D^{m}$ as compared with the standard Stokes-Dirac structure $D$ vanishes, and so in these cases we are back to the standard definition of a distributedparameter port-Hamiltonian system (with $\rho$ being a 1-form, respectively, a 2-form).

Furthermore, if in the 3-dimensional case the 2-form $d v(t)$ happens to be zero at a certain time-instant $t=t_{0}$ (irrotational flow), then it continues to be zero for all time $t \geq t_{0}$. Hence also in this case the extra term (125) in the modified StokesDirac structure $D^{m}$ vanishes, and the port-Hamiltonian system describing the Euler equations reduces to the standard distributed-parameter port-Hamiltonian system given in Definition 4.1.

Remark 6.3. For the modified Stokes-Dirac structure $D^{m}$ given in (124) the space of admissible mappings $K$ adm given in equation (61) is the same as for the StokesDirac structure, but the resulting skew-symmetric bracket has an additional term:

$$
\begin{gathered}
\left\{k^{1}, k^{2}\right\} D^{m}=\int_{Z}\left[\left(\delta_{\rho} k^{1}\right) \wedge(-1)^{r} d\left(\delta_{q} k^{2}\right)+\left(\delta_{q} k^{1}\right) \wedge d\left(\delta_{p} k^{2}\right)\right. \\
\left.+\frac{1}{* \rho} \delta_{v} k^{1} \wedge *\left((* d v) \wedge\left(* \delta_{v} k^{2}\right)\right)\right]-\int_{\partial Z}(-1)^{n-q}\left(\delta_{q} k^{1}\right) \wedge\left(\delta_{p} k^{2}\right)
\end{gathered}
$$

(For the skew-symmetry of the additional term see (125) and Remark 6.1.) 


\section{Conserved quantities and control}

\subsection{Conservation laws of port-Hamiltonian systems}

Let us consider the distributed-parameter port-Hamiltonian system $\Sigma$, as defined in Definition 4.1, on an $n$-dimensional spatial domain $Z$ having state space $\Omega^{p}(Z) \times$ $\Omega^{q}(Z)$ (with $p+q=n+1$ ) and Stokes-Dirac structure $D$ given by (55).

Conservation laws for $\Sigma$, which are independent from the Hamiltonian $H$, are obtained as follows. Let

$$
C: \Omega^{p}(Z) \times \Omega^{q}(Z) \times Z \rightarrow \mathbb{R}
$$

be a function satisfying

$$
d\left(\delta_{p} C\right)=0, \quad d\left(\delta_{q} C\right)=0,
$$

where $d\left(\delta_{p} C\right), d\left(\delta_{q} C\right)$ are defined similarly to (72). Then the time-derivative of $C$ along the trajectories of $\Sigma$ is given as (in view of (146), and using similar calculations as in the proof of Theorem 3.5

$$
\begin{aligned}
\frac{d}{d t} C & =\int_{Z} \delta_{p} C \wedge \dot{\alpha}_{p}+\int_{Z} \delta_{q} C \wedge \dot{\alpha}_{q} \\
& =-\int_{Z} \delta_{p} C \wedge(-1)^{r} d\left(\delta_{q} H\right)-\int_{Z} \delta_{q} C \wedge d\left(\delta_{p} H\right) \\
& =-(-1)^{n-q} \int_{Z} d\left(\delta_{q} H \wedge \delta_{p} C\right)-(-1)^{n-q} \int_{Z} d\left(\delta_{q} C \wedge \delta_{p} H\right) \\
& =\int_{\partial Z} e_{b} \wedge f_{b}^{C}+\int_{\partial Z} e_{b}^{c} \wedge f_{b}
\end{aligned}
$$

where we have denoted, in analogy with (55),

$$
f_{b}^{C}:=\left.\delta_{p} C\right|_{\partial Z}, \quad e_{b}^{C}:=-\left.(-1)^{n-q} \delta_{q} C\right|_{\partial Z}
$$

In particular, if additionally to (146) the function $C$ satisfies

$$
\left.\delta_{p} C\right|_{\partial Z}=0,\left.\quad \delta_{q} C\right|_{\partial Z}=0
$$

then $\frac{d C}{d t}=0$ along the system trajectories of $\Sigma$ for any Hamiltonian $H$. Therefore a function $C$ satisfying (146), (149) is called a Casimir function. If $C$ satisfies (146) but not (149) then $C$ is called a conservation law for $\Sigma$ : its time-derivative is determined by the boundary conditions of $\Sigma$.

Example 7.1. In the case of the telegraph equations (Example 3.2) the total charge

$$
C_{Q}=\int_{0}^{1} Q(t, z) d z
$$

as well as the total magnetic flux

$$
C_{\varphi}=\int_{0}^{1} \varphi(t, z) d z
$$


are both conservation laws. Indeed

$$
\begin{aligned}
\frac{d}{d t} C_{Q} & =-\int_{0}^{1} \frac{\partial I}{\partial z}=I(0)-I(1) \\
\frac{d}{d t} C_{\varphi} & =-\int_{0}^{1} \frac{\partial V}{\partial z} d z=V(0)-V(1)
\end{aligned}
$$

Similarly, in the case of the vibrating string (Example 5.3) conservation laws are $=\int_{0}^{1} \epsilon(t, z) d z=u(t, 1)-u(t, 0)$,

$$
\begin{aligned}
& \frac{d}{d t} \int_{0}^{1} \epsilon(t, z) d z=\frac{d}{d t}(u(t, 1)-u(t, 0))=v(t, 1)-v(t, 0) \\
& \frac{d}{d t} \int_{0}^{1} p(t, z) d z=\sigma(t, 1)-\sigma(t, 0)
\end{aligned}
$$

Conservation laws $C$ for $\Sigma$ which are dependent on the Hamiltonian $H$ are obtained by replacing (146) by the weaker condition

$$
\delta_{q} H \wedge d\left(\delta_{p} C\right)+(-1)^{r} \delta_{p} H \wedge d\left(\delta_{q} C\right)=0
$$

Indeed, it immediately follows from the computation in (147) that under this condition (147) continues to hold.

In the case of the modifies Stokes-Dirac structure $D^{m}$ defined in (124), for any function $C: \Omega^{3}(Z) \times \Omega^{1}(Z) \times Z \rightarrow \mathbb{R}$ satisfying

$$
\delta_{v} H \wedge d\left(\delta_{p} C\right)+\delta_{\rho} H \wedge d\left(\delta_{v} C\right)=0, \quad \rho \in \Omega^{3}(Z), v \in \Omega^{1}(Z)
$$

equation (147) takes the form

$$
\begin{aligned}
\frac{d}{d t} C= & \int_{Z} \delta_{\rho} C \wedge d\left(\delta_{v} H\right)+\int_{Z} \delta_{v} C \wedge\left[d\left(\delta_{\rho} H\right)+\frac{1}{* \rho} *\left((* d v) \wedge\left(* \delta_{v} H\right)\right)\right] \\
= & \int_{\partial Z} \delta_{\rho} C \wedge \delta_{v} H+\int_{\partial Z} \delta_{v} C \wedge \delta_{\rho} H \\
& +\int_{Z} \frac{1}{* \rho} \delta_{v} C *\left((* d v) \wedge\left(* \delta_{v} H\right)\right)
\end{aligned}
$$

Hence we conclude that in order to obtain a conservation law we need to impose an extra condition eliminating the last $\int_{Z}$ integral. A specific example of a conservation law in this case is the helicity

$$
C=\int_{Z} v \wedge d v
$$

with time-derivative

$$
\frac{d}{d t} C=-\int_{\partial Z} f_{b} \wedge d v
$$

A second class of conserved quantities corresponding to the Stokes-Dirac structure $D(55)$ is identified by noting that by (77)

$$
\begin{aligned}
& -d\left(\frac{\partial \alpha_{p}}{\partial t}\right)=(-1)^{r} d\left(d \delta_{q} H\right)=0 \\
& -d\left(\frac{\partial \alpha_{q}}{\partial t}\right)=d\left(d \delta_{p} H\right)=0
\end{aligned}
$$


and thus the differential forms $d \alpha_{p}$ and $d \alpha_{q}$ do not depend on time. Therefore, the component functions of $d \alpha_{p}$ and $d \alpha_{q}$ are conserved quantities of any portHamiltonian system corresponding to $D$.

Example 7.2. In the case of Maxwell's equations (Example 3.1) this yields that $d \mathcal{D}$ and $d \mathcal{B}$ are constant 3 -forms. The 3 -form $d \mathcal{D}$ is the charge density (Gauss' electric law), while by Gauss' magnetic law $d \mathcal{B}$ is actually zero.

In the case of an ideal isentropic fluid (Example 3.4) for which the vorticity $d v\left(t_{0}, z\right)$ is zero at a certain time $t_{0}$ we obtain by the same reasoning (since the additional term in the Stokes-Dirac structure $D^{m}$ is zero for $\left.t_{0}\right)$ that $d v(t, z)$ is zero for all $t \geq t_{0}$ (irrotational flow); cf. Remark 6.2.

\section{Conclusions and final remarks}

In this paper we have exposed a framework for the compositional modelling of distributed-parameter systems, based on our papers [37, 20, 22]. This allows the Hamiltonian formulation of a large class of distributed-parameter systems with boundary energy- ow, including the examples of the telegraph equations, Maxwell's equations, vibrating strings and ideal isentropic fluids. It has been argued that in order to incorporate boundary variables into this formulation the notion of a Dirac structure provides the appropriate generalization of the more commonly used notion of a Poisson structure for evolution equations. The employed Dirac structure is based on Stokes' theorem, and emphasizes the geometrical content of the variables as being differential k-forms. From a physical point of view the Stokes-Dirac structure captures the balance laws inherent to the system, like Faraday's and Ampère's law (in Maxwell's equations), or mass-balance (in the case of an ideal fluid). This situation is quite similar to the lumped-parameter case where the Dirac structure incorporates the topological interconnection laws (Kirchhoff's laws, Newton's third law) and other interconnection constraints (see e.g. [19] [19] [35]). Hence the starting point for the Hamiltonian description is different from the more common approach of deriving Hamiltonian equations from a variational principle and its resulting Lagrangian equations, or (very much related) a Hamiltonian formulation starting from a state space being a co-tangent bundle endowed with its natural symplectic structure. In the case of Maxwell's equations this results in the use of the basic physical variables $D$ and $B$ (the electric and magnetic field inductions), instead of the use of the variable $\mathrm{D}$ (or $\mathrm{E}$ ) together with the vector potential $\mathrm{A}$ (with $d A=B$ ) in the symplectic formulation of Maxwell's equations. It should be of interest to compare both approaches more closely, also in the context of the natural multi-symplectic structures which have been formulated for the Hamiltonian formulation of Lagrangian field equations; see e.g. [5], [15].

A prominent and favorable property of Dirac structures is that they are closed under power-conserving interconnection. This has been formally proven in the finitedimensional case, but the result carries through to the infinite-dimensional case as well. It is a property of fundamental importance since it enables to link portHamiltonian systems (lumped- or distributed-parameter) to each other to obtain an interconnected port-Hamiltonian system with total energy being the sum of the 
Hamiltonians of its constituent parts. Clearly, this is equally important in modelling (coupling e.g. solid components with fluid components, or finite-dimensional electric components with transmission lines), as in control. First of all, it enables to formulate directly distributed-parameter systems with constraints as (implicit) Hamiltonian systems, like this has been done in the finite-dimensional case for mechanical systems with kinematic constraints, multi-body systems, and general electrical networks. Secondly, from the control perspective the notion of feedback control can be understood on its most basic level as the coupling of given physical components with additional control components (being themselves physical systems, or software components linked to sensors and actuators). A preliminary study from this point of view of a control scheme involving transmission lines has been provided in [30]. Among others, this opens up the way for the application of passivity-based control techniques, which have been proven to be very effective for the control of lumpedparameter physical systems modelled as port-Hamiltonian systems.

\section{References}

[1] Ralph Abraham and Jerrold E. Marsden. Foundations of Mechanics. Addison, ii edition, March 1994. ISBN 0-8053-0102-X.

[2] A.M.Bloch and P.E.Crouch. Representation of Dirac structures on vector spaces and nonlinear lcv-circuits. In H.Hermes G. Ferraya, R.Gardner and H.Sussman, editors, Proc. of Symposia in Pure mathematics, Differential Geometry and Control Theory, volume 64, pages 103-117, 1999.

[3] A.J. van der Schaft G. Blankenstein. Symmetry and reduction in implicit generalized hamiltonian systems. Rep. Math. Phys., 47:57-100, 2001.

[4] P.C. Breedveld. Physical Systems Theory in Terms of Bond Graphs. PhD thesis, Technische Hogeschool Twente, Enschede, The Netherlands, feb 1984. ISBN 90-90005999-4.

[5] T.J. Courant. Dirac manifolds. Trans. American Math. Soc. 319, pages 631661, 1990.

[6] M. Dalsmo and A.J. van der Schaft. On representations and integrability of mathematical structures in energy-conserving physical systems. SIAM Journal of Control and Optimization, 37(1):54-91, 1999.

[7] I. Dorfman. Dirac structures and integrability of nonlinear evolution equations. John Wiley, 1993.

[8] D. Eberard and B.M. Maschke. An extension of port Hamiltonian systems to irreversible systems. In Proc. Int. Conf. on Nonlinear Systems' Theory and Control, NOLCOS'04, Stuttgart, Germany, 2004. submitted.

[9] H.O. Fattorini. Boundary control systems. SIAM J. Control and Opt., (6):349$385,1968$. 
[10] E. Godlewsky and P. Raviart. Numerical Approximation of Hyperbolic Systems of Conservation Laws, volume 118 of Applied Mathematical Sciences. Springer Verlag, New-York, USA, 1996.

[11] T.E. Ratiu A. Weinstein D.D. Holm, J.E. Marsden. Nonlinear stability of fluid and plasma equilibria. Phys.Rep., 123:1-116, 1985.

[12] A. Jamiolkowski R.S. Ingarden. Classical Electrodynamics. PWN-Polish Sc. Publ. Elsevier, Warszawa, Poland, 1985.

[13] Dean C. Karnopp, Donald L. Margolis, and Ronald C. Rosenberg. System Dynamics, A Unified Approach. Wiley, 1990.

[14] B.A. Khesin V. I. Arnold. Topological Methods in Hydrodynamics, volume 125 of Applied Mathematical Sciences. Springer Verlag, New York, USA, 1998.

[15] Y. Le Gorrec, H. Zwart, B.M. Maschke. in preparation.

[16] Paulette Libermann and Charles-Michel Marle. Symplectic Geometry and Analytical Mechanics. D. Reidel Publishing Company, Dordrecht, Holland, 1987. ISBN 90-277-2438-5.

[17] A. Weinstein J.E. Marsden, T. Ratiu. Reduction and Hamiltonian structures on duals of semidirect product Lie algebras. AMS Contemporary Mathematics, 28:55-100, 1984.

[18] A. Weinstein J.E. Marsden, T. Ratiu. Semidirect products and reduction in Mechanics. Trans. American Math. Society, (281):147-177, 1984.

[19] B.M. Maschke and A.J. van der Schaft. Modelling and Control of Mechanical Systems, chapter Interconnected Mechanical systems. Part 1 and 2, pages 1-30. Imperial College Press, London, 1997. ISBN 1-86094-058-7.

[20] B.M. Maschke and A.J. van der Schaft. Port controlled Hamiltonian representation of distributed parameter systems. In R. Ortega N.E. Leonard, editor, Proc. IFAC Workshop on modeling and Control of Lagrangian and Hamiltonian Systems, Princeton, USA, 2000.

[21] A.J. van der Schaft \& G. Escobar B.M. Maschke, R. Ortega. An energy-based derivation of lyapunov functions for forced systems with application to stabilizing control. In Proc. 14th IFAC World Congress, volume E, pages 409-414, Beijing, China, July 1999.

[22] A.J. van der Schaft B.M. Maschke. Nonlinear Control in the Year 2000, chapter Hamiltonian representation of distributed parameter systems with boundary energy flow, pages 137-142. Springer-Verlag, eds. a. isidori, f. lamnabhi-lagarrigue, w. respondek edition, 2000.

[23] B.M. Maschke, A.J. van der Schaft, and P.C. Breedveld. An intrinsic Hamiltonian formulation of network dynamics: Non-standard poisson structures and gyrators. Journal of the Franklin institute, 329(5):923-966, 1992. Printed in Great Britain. 
[24] P.J. Morrison \& J.M. Greene. Noncanonical Hamiltonian density formulation of hydrodynamics and ideal magnetohydrodynamics. Phys. Rev. Letters, (45):790$794,1980$.

[25] P.J. Morrison. Hamiltonian description of the ideal fluid. Rev. Mod. Phys., (70):467-521, 1998.

[26] Peter J. Olver. Applications of Lie Groups to Differential Equations, volume 107 of Graduate texts in mathematics. Springer, New-York, ii edition, 1993. ISBN 0-387-94007-3.

[27] R. Ortega A.J. van der Schaft I. Mareels B.Maschke. Putting energy back in control. IEEE Control Systems Magazine, 21(2):18- 32, April 2001.

[28] B. Maschke R. Ortega, A.J. van der Schaft and G. Escobar. Interconnection and damping assignment: passivity-based control of port-controlled Hamiltonian systems. Automatica, 38:585-596, 2002.

[29] I. Prigogine. Introduction to Thermodynamics of Irreversible Processes. John Wiley and Sons, 1962.

[30] H. Rodriguez, A.J. van der Schaft, R. Ortega. On stabilization of nonlinear distributed parameter port-controlled Hamiltonian systems via energy shaping. Proc. 40th IEEE Conf. Decision and Control (CDC), Orlando FL 2001, pp.131136.

[31] D. Serre. Systems of Conservation Laws. Cambridge University Press, Cambridge, U.K., 1999.

[32] S. Stramigioli, B.M. Maschke, and A.J. van der Schaft. Passive output feedback and port interconnection. In Proc NOLCOS 1998, Enschede, The Netherlands, July 1998.

[33] A.J. van der Schaft. Implicit Hamiltonian systems with symmetry. Reports on Mathematical Physics, 41:203-221, 1994.

[34] A.J. van der Schaft. $L_{2}$-Gain and Passivity Techniques in Nonlinear Control. Springer Communications and Control Engineering series. Springer-Verlag, London, 2nd revised and enlarged edition, 2000. first edition Lect. Notes in Control and Inf. Sciences, vol. 218, Springer-Verlag, Berlin, 1996.

[35] A.J. van der Schaft and B.M. Maschke. The Hamiltonian formulation of energy conserving physical systems with external ports. Archiv für Elektronik und Übertragungstechnik, 49(5/6):362-371, 1995.

[36] A.J. van der Schaft and B.M. Maschke Fluid dynamical systems as hamiltonian boundary control systems. In Proc. 40th IEEE Conf. on Decision and Control, pages 4497-4502, Orlando, USA, Dec. 2001. 
[37] A.J. van der Schaft and B.M. Maschke. Hamiltonian formulation of distributed parameter systems with boundary energy flow. J. of Geometry and Physics, 42:166-174, 2002. 